\title{
A MULTILEVEL MONTE CARLO FINITE DIFFERENCE METHOD FOR RANDOM SCALAR DEGENERATE CONVECTION DIFFUSION EQUATIONS
}

\author{
Ujjwal Koley \\ Tata Institute of Fundamental Research, \\ Centre For Applicable Mathematics, \\ Post Bag No. 6503, GKVK Post Office, Sharada Nagar, Chikkabommasandra, \\ Bangalore 560065, India. \\ ujjwal@math.tifrbng.res.in \\ Nils Henrik Risebro* \\ Department of Mathematics, University of Oslo \\ P.O. Box 1053, Blindern \\ N-0316 Oslo, Norway. \\ nilshr@math.uio.no \\ Christoph Schwab ${ }^{\dagger}$ \\ Seminar for Applied Mathematics, ETH Zürich \\ Rämistrasse 101, Zürich, Switzerland. \\ schwab@sam.math.ethz.ch \\ Franziska Weber ${ }^{\ddagger}$ \\ Seminar for Applied Mathematics,ETH Zürich \\ Rämistrasse 101, Zürich, Switzerland. \\ franziska.weber@sam.math.ethz.ch
}

\begin{abstract}
This paper proposes a Finite Difference Multilevel Monte Carlo algorithm for degenerate parabolic convection diffusion equations where the convective and diffusive fluxes are allowed to be random. We establish a notion of stochastic entropy solutions to these. Our chief goal is to efficiently compute approximations to statistical moments of these stochastic entropy solutions. To this end we design a multilevel Monte Carlo method based on a finite volume scheme for each sample. We present a novel convergence rate analysis of the combined multilevel Monte Carlo Finite Volume method, allowing in particular for low $p$-integrability of the random solution with $1<p \leq 2$, and low deterministic convergence rates (here, the theoretical rate is $1 / 3$ ). We analyze the design and error versus work of the multilevel estimators. We obtain that the maximal rate (based on optimizing possibly the pessimistic upper bounds on the discretization error), is obtained for $p=2$, for finite volume convergence rate of $1 / 3$. We conclude with numerical experiments.
\end{abstract}

* Risebro supported in part by NFR project 214495 LIQCRY

${ }^{\dagger}$ Schwab supported in part by ERC AdG247277 STAHDPDE

${ }^{\ddagger}$ Weber supported in part by NFR project 214495 LIQCRY and by ERC STG 306279 SPARCCLE 


\section{Introduction}

Many problems in physics and engineering are modeled by nonlinear, possibly strongly degenerate, convection diffusion equation. The Cauchy problem for such equations takes the form

$$
\begin{cases}u_{t}+\operatorname{div} f(u)=\Delta A(u), & (x, t) \in \Pi_{T}, \\ u(0, x)=u_{0}(x), & x \in \mathbb{R}^{d},\end{cases}
$$

where $\Pi_{T}=\mathbb{R}^{d} \times(0, T)$ with $T>0$ fixed, $u: \Pi_{T} \rightarrow \mathbb{R}$ is the unknown function, $f=\left(f_{1}, \ldots, f_{d}\right)$ is the flux function, and $A$ is the nonlinear diffusion. Regarding this, the basic assumption is that $a(u):=A^{\prime}(u) \geq 0$, for all $u$. When (1.1) is nondegenerate, i.e., $a(u)>0$, it is well known that (1.1) admits a unique classical solution [33]. This contrasts with the degenerate case where $a(u)$ may vanish for some values of $u$. A simple example of a degenerate equation is the porous medium equation

$$
u_{t}=\Delta\left(u^{m}\right), m>1,
$$

which degenerates at $u=0$. This equation has served as a simple model to describe processes involving fluid flow, heat transfer or diffusion. Examples of applications are in the description of the flow of an isentropic gas through a porous medium, modelled by Leibenzon [27] and Muskat [31] around 1930, in the study of groundwater flow by Boussisnesq in 1903 [3] or in heat radiation in plasmas, Zel'dovich and collaborators around 1950, [39]. In general, a manifestation of the degeneracy in (1.1) is the finite speed of propagation of disturbances. If $a(0)=0$, and if at some fixed time the solution $u$ has compact support, then it will continue to have compact support for all later times.

By the term "strongly degenerate" we mean that there is an open interval such that $a(u)=0$ if $u$ is in this interval. Hence, the class of equations under consideration is very large and contains the heat equation, the porous medium equation and scalar conservation laws. Independently of the smoothness of the initial data, due to the degeneracy of the diffusion, singularities may form in the solution $u$. Therefore we consider weak solutions which are defined as follows.

Definition 1.1. Set $\Pi_{T}=\mathbb{R}^{d} \times(0, T)$. A function

$$
u(t, x) \in C\left([0, T] ; L^{1}\left(\mathbb{R}^{d}\right)\right) \cap L^{\infty}\left(\Pi_{T}\right)
$$

is a weak solution of the initial value problem (1.1) if it satisfies:

D. $1 \operatorname{grad} A(u) \in L^{\infty}\left(\Pi_{T}\right)$.

D.2 For all test functions $\varphi \in \mathcal{D}\left(\mathbb{R}^{d} \times[0, T)\right)$

$$
\iint_{\Pi_{T}}\left(u \varphi_{t}+f(u) \cdot \operatorname{grad} \varphi+A(u) \Delta \varphi\right) d x d t+\int_{\mathbb{R}^{d}} u_{0}(x) \varphi(x, 0) d x=0 .
$$


In view of the existence theory, the condition D.1 is natural, and thanks to this we can replace (1.2) by

$$
\iint_{\Pi_{T}} u \varphi_{t}+(f(u)-\operatorname{grad} A(u)) \cdot \operatorname{grad} \varphi d x d t+\int_{\mathbb{R}^{d}} u_{0}(x) \varphi(x, 0) d x=0 .
$$

If $A$ is constant on a whole interval, then weak solutions are not uniquely determined by their initial data, and one must impose an additional entropy condition to single out the physically relevant solution. A weak solution satisfies the entropy condition if

$$
\varrho(u)_{t}+\operatorname{div} q(u)-\Delta r(u) \leq 0 \text { in } \mathcal{D}^{\prime}\left(\Pi_{T}\right),
$$

for all convex, twice differentiable functions $\varrho: \mathbb{R} \rightarrow \mathbb{R}$, where $q$ and $r$ are defined by

$$
q^{\prime}(u)=\varrho^{\prime}(u) f^{\prime}(u), \text { and } r^{\prime}(u)=\varrho^{\prime}(u) A^{\prime}(u) .
$$

Via a standard limiting argument this implies that (1.3) holds for the Kružkov entropies $\varrho(u)=|u-c|$ for all constants $c$. We call a weak solution satisfying the entropy condition an entropy solution.

For scalar conservation laws, the entropy framework (usually called entropy conditions) was introduced by Kružkov [24] and Vol'pert [37], while for degenerate parabolic equations entropy solution were first considered by Vol'pert and Hudajev [38]. Uniqueness of entropy solutions to (1.1) was first proved by Carrillo [4].

Over the years, there has been a growing interest in numerical approximation of entropy solutions to degenerate parabolic equations. Finite difference and finite volume schemes for degenerate equations were analysed by Evje and Karlsen $[11,10,9,12]$ (using upwind difference schemes), Holden et al. [18,19] (using operator splitting methods), Kurganov and Tadmor [25] (central difference schemes), Bouchut et al. [2] (kinetic BGK schemes), Afif and Amaziane [1] and Ohlberger, Gallouët et al. [32,14,15] (finite volume methods), Cockburn and Shu [7] (discontinuous Galerkin methods) and Karlsen and Risebro [23,22] (monotone difference schemes). Many of the above papers show that the approximate solutions converge to the unique entropy solution as the discretization parameter vanishes. Rigorous estimates of the convergence rate of finite volume schemes for degenerate parabolic equations were proved in [20] (1-d) and [21] (multi-d).

This classical paradigm for designing efficient numerical schemes assumes that data for (1.1), i.e., initial data $u_{0}$, convective flux and diffusive flux are known exactly.

In many situations of practical interest, however, these data are not known exactly due to inherent uncertainty in modelling and measurements of physical parameters such as, for example, the specific heats in the equation of state for compressible gases, or the relative permeabilities in models of multi-phase flow in porous media. Often, the initial data are known only up to certain statistical quantities of interest like the mean, variance, higher moments, and in some cases, 
the law of the stochastic initial data. In such cases, a mathematical formulation of (1.1) is required which allows for random data. The problem of random initial data was considered in [29], and the existence and uniqueness of a random entropy solution was shown, and a convergence analysis for Multilevel Monte Carlo Finite volume discretizations (MLMCFV) was given. The MLMC discretization of balance laws with random source terms was investigated in [30].

The first aim of this paper is to extend this mathematical framework to include degenerate convection diffusion equations with random convective and diffusive flux functions with possibly correlated random perturbations. We define random entropy solutions and provide an existence and uniqueness result, generalizing the classical well-posedness results to the case of uncertain initial data, and flux functions.

The second aim of this paper is to design fast and robust numerical algorithms for computing random entropy solutions. In particular, we focus on statistical sampling techniques of the Monte Carlo (MC) type. However, since the rate of convergence of MC method is $1 / 2$, we propose a MLMCFD method based on implicit/explicit finite difference schemes for deterministic convection diffusion equations. In particular, we show that MLMCFD schemes converge. Moreover, we use a Lagrange multiplier type argument to determine the optimal number of $\mathrm{MC}$ samples needed to minimize the computational work.

The rest of the paper is organized as follows: In Section 2, we present the probabilistic framework used in this paper. In particular, we review notions of random variables taking values in separable Banach spaces. Section 3 is devoted to a review of convergence rates from $[20,21]$ on convergence rates for scalar degenerate deterministic convection diffusion problems. Particular attention is paid to the definition of entropy solutions and to existence-, uniqueness- and continuous dependence results, and to the definition of the random entropy solutions, and to sufficient conditions ensuring their measurability and integrability. In Section 4, we address the discretization of such underlying problems. First, again reviewing convergence rates of FD schemes for the deterministic case from [20,21], which we then extend to $\mathrm{MC}$ as well as MLMC versions for the degenerate convection diffusion problem with random coefficients and flux functions. The final Section 5 is then devoted to numerical experiments which confirm the theoretical convergence estimates and, in fact, indicate that they probably are pessimistic, at least in the particular test problems considered.

\section{Preliminaries from Probability Theory}

To set the notation, we recapitulate prerequistes from measure and probability theory which are needed in the subsequent sections. For proofs and further details, we refer for example to [36, Chapter 1] and to the references there. 


\subsection{Random variables on Banach spaces}

Let $(\Omega, \mathcal{F}, \mathbb{P})$ be a probability space, and let $(E, \mathcal{B}(E))$ be a Banach space $E$ with its Borel $\sigma$-algebra $\mathcal{B}(E)$. A map $G: \Omega \rightarrow E$ is called a $\mathbb{P}$-simple function if it is of the form

$$
G(\omega)=\sum_{j=1}^{J} g_{j} \mathbb{1}_{A_{j}}(\omega), \text { where } \mathbb{1}_{A}(\omega)= \begin{cases}1 & \omega \in A, \\ 0 & \text { otherwise }\end{cases}
$$

and $g_{j} \in E$ for $j=1, \ldots, J$, for some finite $J$ and for $A_{j} \in \mathcal{F}$. A map $f: \Omega \rightarrow E$ is strongly $\mathcal{F}$-measurable if there exists a sequence of simple functions $f_{n}$ converging to $f$ (in the norm of $E$ ) $\mathbb{P}$-almost everywhere on $\Omega$.

We call two strongly $\mathbb{P}$-measurable functions $f, g: \Omega \rightarrow E$ which agree $\mathbb{P}$-almost everywhere on $\Omega, \mathbb{P}$-versions of each other. We shall need the following lemma.

Lemma 2.1. [36, Corollary 1.13] Let $E_{1}$ and $E_{2}$ be Banach spaces, and $(\Omega, \mathcal{F}, \mathbb{P})$ a probability space. If $f: \Omega \rightarrow E_{1}$ is strongly measurable, and $\phi: E_{1} \rightarrow E_{2}$ is continuous, then the composition $\phi \circ f: \Omega \rightarrow E_{2}$ is strongly measurable.

Next, we define the integral of a simple function $G=\sum g_{j} \mathbb{1}_{A_{j}}$ by

$$
\int_{\Omega} G d \mathbb{P}=\sum_{j=1}^{N} g_{j} \mathbb{P}\left(A_{j}\right) .
$$

If $f: \Omega \rightarrow E$ is strongly measurable, we say that $f$ is Bochner integrable if there exists a sequence of simple functions $\left\{f_{n}\right\}_{n \geq 0}$ converging to $f \mathbb{P}$-almost everywhere, and

$$
\lim _{n \rightarrow \infty} \int_{\Omega}\left\|f-f_{n}\right\|_{E} d \mathbb{P}=0,
$$

([36, Def. 1.15]). We then define the Bochner integral of $f$ by

$$
\int_{\Omega} f d \mathbb{P}:=\lim _{n \rightarrow \infty} \int_{\Omega} f_{n} d \mathbb{P} .
$$

A strongly measurable function $f: \Omega \rightarrow E$ is Bochner integrable if and only if

$$
\int_{\Omega}\|f\|_{E} d \mathbb{P}<\infty
$$

(see for example [36, Prop. 1.16]) in which case

$$
\left\|\int_{\Omega} f d \mathbb{P}\right\|_{E} \leq \int_{\Omega}\|f\|_{E} d \mathbb{P}
$$

For each $1 \leq p<\infty$ we can define the Banach spaces $L^{p}(\Omega ; E)$ to consist of those strongly measurable functions $f$ for which the integrals

$$
\int_{\Omega}\|f\|_{E}^{p} d \mathbb{P}<\infty
$$


These spaces have the natural norm

$$
\|f\|_{L^{p}(\Omega ; E)}=\left(\int_{\Omega}\|f\|_{E}^{p} d \mathbb{P}\right)^{1 / p}
$$

If $p=\infty$, we define $L^{\infty}(\Omega ; E)$ to be the space of strongly measurable functions $f: \Omega \rightarrow E$ for which there exists a number $r \geq 0$ such that $\mathbb{P}\left(\|f\|_{E}>r\right)=0$. Together with the norm

$$
\|f\|_{L^{\infty}(\Omega ; E)}:=\inf \left\{r \geq 0: \mathbb{P}\left(\|f\|_{E}>r\right)=0\right\},
$$

this space is a Banach space as well.

If $f: \Omega \rightarrow E$ is strongly measurable and $(\Omega, \mathcal{F}, \mathbb{P})$ is a probability space, we call $f$ an $E$-valued random variable.

In the following, we will be interested in random variables $X: \Omega \rightarrow E_{j}, j=1,2$, mapping from some probability space $(\Omega, \mathcal{F}, P)$ into subsets of the Banach spaces $E_{j}, j=1,2$, equipped with the Borel $\sigma$-algebra $\mathcal{B}\left(E_{j}\right)$, where $E_{1}=L^{1}\left(\mathbb{R}^{d}\right) \times$ $W^{1, \infty}(I) \times W^{1, \infty}(I)$, for a closed and bounded interval $I=\left[M_{-}, M_{+}\right] \subset(-\infty, \infty)$, $-\infty<M_{-}<M_{+}<\infty$, and $E_{2}=C\left([0, T] ; L^{1}\left(\mathbb{R}^{d}\right)\right), T>0$. On $W^{1, \infty}(I)$, we choose the norm

$$
\|f\|_{W^{1, \infty}(I)}=\underset{x \in I}{\operatorname{ess} \sup }|f(x)|+\underset{x \in I}{\operatorname{ess} \sup _{1}}\left|f^{\prime}(x)\right|, \quad f \in W^{1, \infty}(I) .
$$

On $E_{1}$, we will use the sum norm

$$
\|\mathbf{g}\|_{E_{1}}=\left\|g_{1}\right\|_{L^{1}\left(\mathbb{R}^{d}\right)}+\left\|g_{2}\right\|_{W^{1, \infty}(I)}+\left\|g_{3}\right\|_{W^{1, \infty}(I)}, \quad \mathbf{g}=\left(g_{1}, g_{2}, g_{3}\right) \in E_{1},
$$

and on $E_{2}$, the norm

$$
\|h\|_{E_{2}}=\sup _{0 \leq t \leq T} \int_{\mathbb{R}}|h(t, x)| d x, \quad h \in E_{2} .
$$

\subsection{Approximation of moments of random variables on Banach spaces}

Often, one is not interested in the law of a random variable $X: \Omega \rightarrow E_{1}$ on a Banach space $E_{1}$, but only in statistics, such as the mean field (ensemble average) $\mathbb{E}[Y]$ of quantities of interest $Y=g(X)$ of it (for some continuous mapping $g: E_{1} \rightarrow E_{2}, E_{2}$ another Banach space). As explicit expressions for those are not always available, one has to approximate them. This can be done using Monte Carlo sampling. To this end, let $\widehat{Y}_{i}:=g\left(\widehat{X}_{i}\right): \Omega \rightarrow E_{2}, i=1, \ldots, M$, be independent identically distributed random variables. We define the the sample average

$$
E_{M}[Y]:=\frac{1}{M} \sum_{i=1}^{M} \widehat{Y}_{i},
$$

as so-called Monte Carlo estimator for $\mathbb{E}[Y]$. We would like to know how good of an estimate the sample average $E_{M}[Y]$ is for the expectation $\mathbb{E}[Y]$ of $Y$. Specifically, we are interested in at what rate

$$
\mathbb{E}\left[\left\|\mathbb{E}[Y]-E_{M}[Y]\right\|_{E_{2}}^{p}\right]^{1 / p}
$$


converges as $M \rightarrow \infty$ for some $1 \leq p<\infty$. If $p=2$ and if $E$ is a Hilbert space then it is classical that the so called mean square error (MSE) satisfies

$$
\mathbb{E}\left[\left\|\mathbb{E}[Y]-E_{M}[Y]\right\|_{E}^{2}\right]=\frac{1}{M} \mathbb{E}\left[\|Y-\mathbb{E}[Y]\|_{E}^{2}\right]=\frac{1}{M} \operatorname{Var}[Y] .
$$

Equation (2.4) is only meaningful for Hilbert space valued random variables. For general Banach spaces, the convergence rate depends on the type of the Banach space, [26, Page 246].

Definition 2.2. Let $1 \leq q \leq \infty$ and $Z_{j}, j \in \mathbb{N}$ a sequence of independent Rademacher random variables. A Banach space $E$ is said to be of type $q \geq 1$ if there is a type constant $C_{t}>0$ such that for all finite sequences $\left(x_{j}\right)_{j=1}^{N} \subset E$, $N \in \mathbb{N}$,

$$
\left\|\sum_{j=1}^{N} Z_{j} x_{j}\right\|_{E} \leq C_{t}\left(\sum_{j=1}^{N}\left\|x_{j}\right\|_{E}^{q}\right)^{1 / q} .
$$

Remark 2.3. (i) By the triangle inequality, every Banach space has type 1. (ii) Hilbert spaces (and in particular finite-dimensional spaces) have type 2 (with the type constant $C_{t}$ depending on the dimension, in general) (iii) $L^{p}$-spaces have type $q=\min \{2, p\}$ for $1 \leq p<\infty,[26$, Page 247].

One has the following result [26, Proposition 9.11] for Banach spaces of type $q$ :

Proposition 2.4. Let $E$ be a Banach space of type $q$ with type constant $C_{t}$. Then, for every finite sequence $\left(Y_{j}\right)_{j=1}^{M}$ of independent random variables in $L^{q}(\Omega ; E)$ with zero mean, one has,

$$
\mathbb{E}\left[\left\|\sum_{j=1}^{N} Y_{j}\right\|_{E}^{q}\right] \leq\left(2 C_{t}\right)^{q} \sum_{j=1}^{N} \mathbb{E}\left[\left\|Y_{j}\right\|_{E}^{q}\right] .
$$

This implies a convergence rate in $L^{q}(\Omega)$ for the Monte Carlo estimator (2.3).

Corollary 2.5. Let $E$ be a Banach space of type $q$ with a type constant $C_{t}$. Then for every finite sequence $\left(Y_{j}\right)_{j=1}^{N}$ of iid random variables with zero mean and with $Y_{j}(\omega) \sim Y(\omega)$ in $L^{q}(\Omega)$ and $Y \in L^{q}(\Omega ; E)$, there holds

$$
\mathbb{E}\left[\left\|E_{M}[Y]\right\|_{E}^{q}\right]=\mathbb{E}\left[\left\|\frac{1}{M} \sum_{j=1}^{M} Y_{j}\right\|_{E}^{q}\right] \leq\left(2 C_{t}\right)^{q} M^{1-q} \mathbb{E}\left[\|Y\|_{E}^{q}\right] .
$$

For $q=2$, we recover (2.4) (up to the value of the constant).

\section{Degenerate Convection Diffusion Equation with Random Diffusive Flux}

We develop a theory of random entropy solutions for degenerate convection diffusion equation with a class of random flux flunctions, proving in particular the existence 
and uniqueness of a random entropy solution. To this end, we first review classical results on degenerate convection diffusion equation with deterministic data.

\subsection{Deterministic Scalar Degenerate Convection Diffusion Equation}

We consider the Cauchy problem for degenerate convection diffusion equation of the form

$$
\begin{cases}u_{t}+\operatorname{div} f(u)=\operatorname{div}(a(u) \operatorname{grad} u), & (x, t) \in \Pi_{T}, \\ u(0, x)=u_{0}(x), & x \in \mathbb{R}^{d},\end{cases}
$$

\subsection{Entropy Solutions}

It is well-known that if $f$ is Lipschitz continuous and $a(u) \geq 0$, then the deterministic Cauchy problem (3.1) admits, for each $u_{0} \in L^{1}\left(\mathbb{R}^{d}\right) \cap L^{\infty}\left(\mathbb{R}^{d}\right)$, a unique entropy solution (see, e.g., $[16,35,8])$. Moreover, for every $t>0, u(\cdot, t) \in L^{1}\left(\mathbb{R}^{d}\right) \cap L^{\infty}\left(\mathbb{R}^{d}\right)$ and several properties of the (nonlinear) data-to-solution operator

$$
S:\left(u_{0}, f, A\right) \longmapsto u(\cdot, t)=S(t)\left(u_{0}, f, A\right), \quad t>0,
$$

will be crucial for our subsequent development. To state these properties of $\{S(t)\}_{t \geq 0}$, following [11], we introduce the set of admissible initial data

$$
\mathcal{A}(f, A):=\left\{z \in L^{1}\left(\mathbb{R}^{d}\right) \cap B V\left(\mathbb{R}^{d}\right)|| f(z)-\left.\operatorname{grad} A(z)\right|_{B V}<\infty\right\} .
$$

Next, we collect fundamental results regarding the entropy solution $u$ of (3.1) in the following theorem, for a proof see $[38,5]$,

Theorem 3.1. Let $f$ and $A$ be locally Lipschitz continuous functions. Then

1) For every $u_{0} \in \mathcal{A}(f, A)$, the initial value problem (3.1) admits a unique $B V$ entropy weak solution $u \in C\left([0, T] ; L_{\mathrm{loc}}^{1}\left(\mathbb{R}^{d}\right)\right)$.

2) For every $t>0$, the (nonlinear) data-to-solution map $S(t)$ given by

$$
u(\cdot, t)=S(t)\left(u_{0}, f, A\right)
$$

satisfies

i) For fixed $f, A \in \operatorname{Lip}(\mathbb{R}), S(t)(\cdot, f, A): L_{\mathrm{loc}}^{1}\left(\mathbb{R}^{d}\right) \rightarrow L^{1}\left(\mathbb{R}^{d}\right)$ is a (nonexpansive) Lipschitz map, i.e.,

$$
\left\|S(t)\left(u_{0}, f, A\right)-S(t)\left(v_{0}, f, A\right)\right\|_{L^{1}\left(\mathbb{R}^{d}\right)} \leq\left\|u_{0}-v_{0}\right\|_{L^{1}\left(\mathbb{R}^{d}\right)} .
$$

ii) For every $u_{0} \in \mathcal{A}(f, A), f, A \in \operatorname{Lip}_{\mathrm{loc}}(\mathbb{R})$

$$
\begin{aligned}
\left\|S(t)\left(u_{0}, f, A\right)\right\|_{L^{\infty}\left(\mathbb{R}^{d}\right)} & \leq\left\|u_{0}\right\|_{L^{\infty}\left(\mathbb{R}^{d}\right)} \\
\left\|S(t)\left(u_{0}, f, A\right)\right\|_{L^{1}\left(\mathbb{R}^{d}\right)} & \leq\left\|u_{0}\right\|_{L^{1}\left(\mathbb{R}^{d}\right)} \\
\left\|S(t)\left(u_{0}, f, A\right)\right\|_{B V\left(\mathbb{R}^{d}\right)} & \leq\left\|u_{0}\right\|_{B V\left(\mathbb{R}^{d}\right)} \\
|f(u(\cdot, t))-\operatorname{grad} A(u(\cdot, t))|_{B V\left(\mathbb{R}^{d}\right)} & \leq\left|f\left(u_{0}\right)-\operatorname{grad} A\left(u_{0}\right)\right|_{B V\left(\mathbb{R}^{d}\right)} .
\end{aligned}
$$


iii) Lipschitz continuity in time: For any $t_{1}, t_{2}>0, u_{0} \in \mathcal{A}(f, A)$,

$$
\begin{aligned}
& \left\|S\left(t_{1}\right)\left(u_{0}, f, A\right)-S\left(t_{2}\right)\left(u_{0}, f, A\right)\right\|_{L^{1}\left(\mathbb{R}^{d}\right)} \\
& \quad \leq\left|f\left(u_{0}\right)-\operatorname{grad} A\left(u_{0}\right)\right|_{B V\left(\mathbb{R}^{d}\right)}\left|t_{1}-t_{2}\right| .
\end{aligned}
$$

Proof. Point 1) of Theorem 3.1 is proved in [38] or [5, Thm 1.1], (3.3), (3.5) also follow from [5, Thm 1.1], (3.4) was proved in [5, Thm 1.2], and (3.6), (3.7), (3.8) were proved in [38].

Remark 3.2. We can use the entropy condition (1.3) to obtain $L^{p}\left(\mathbb{R}^{d}\right)$-estimates on the solution at time $t$. Let $\rho_{\varepsilon}$ be a smooth, sign-preserving approximation of the function $\rho(u)=|u|^{p}$ for $1 \leq p<\infty$, then (1.3) implies after integrating in space and time

$$
\int_{\mathbb{R}^{d}} \rho_{\varepsilon}(u(t, x)) d x \leq \int_{\mathbb{R}^{d}} \rho_{\varepsilon}\left(u_{0}(x)\right) d x .
$$

Letting $\varepsilon \rightarrow 0$, we obtain

$$
\|u(t, \cdot)\|_{L^{p}\left(\mathbb{R}^{d}\right)}^{p} \leq\left\|u_{0}\right\|_{L^{p}\left(\mathbb{R}^{d}\right)}^{p} .
$$

In our convergence analysis of MC-FD discretizations of degenerate convection diffusion equation with random fluxes, we will need the following result regarding continuous dependence of $S$ with respect to $f$ and $A$ ([6, Thm. 3]):

Theorem 3.3. Assume $u_{0}, v_{0} \in B V\left(\mathbb{R}^{d}\right) \cap L^{1}\left(\mathbb{R}^{d}\right) \cap L^{\infty}\left(\mathbb{R}^{d}\right)$, and $f(\cdot), g(\cdot), A(\cdot)$, $B(\cdot) \in \operatorname{Lip}_{\text {loc }}(\mathbb{R})$ with $A^{\prime}, B^{\prime} \geq 0$.

Then the unique entropy solutions $u(t, \cdot)=S(t)\left(u_{0}, f, A\right)$ and $v(t, \cdot)=$ $S(t)\left(v_{0}, g, B\right)$ of (3.1) with initial data $u_{0}, v_{0}$, convective flux functions $f$ and $g$ and with diffusive flux functions $A$ and $B$ satisfy the Kružkov entropy conditions, and the à priori continuity estimate

$$
\begin{aligned}
\|u(\cdot, t)-v(\cdot, t)\|_{L^{1}\left(\mathbb{R}^{d}\right)} & \leq\left\|u_{0}-v_{0}\right\|_{L^{1}\left(\mathbb{R}^{d}\right)} \\
& +C\left(t\left\|f^{\prime}-g^{\prime}\right\|_{L^{\infty}\left(M_{-}, M_{+}\right)}+4 \sqrt{t}\left\|\sqrt{A^{\prime}}-\sqrt{B^{\prime}}\right\|_{L^{\infty}\left(M_{-}, M_{+}\right)}\right),
\end{aligned}
$$

where $M_{-} \leq u_{0} \leq M_{+}$and $C=\left|u_{0}\right|_{B V\left(\mathbb{R}^{d}\right)}<\infty$. The above estimate holds for every $0 \leq t \leq T$.

Remark 3.4. Using that for nonnegative numbers $a, b \geq 0, a \neq 0$,

$$
|\sqrt{a}-\sqrt{b}|=\sqrt{|a-b|} \frac{\sqrt{|a-b|}}{\sqrt{a}+\sqrt{b}} \leq \sqrt{|a-b|},
$$

it follows from (3.10) that under the assumptions of Theorem 3.3,

$$
\begin{aligned}
\|u(\cdot, t)-v(\cdot, t)\|_{L^{1}\left(\mathbb{R}^{d}\right)} & \leq\left\|u_{0}-v_{0}\right\|_{L^{1}\left(\mathbb{R}^{d}\right)} \\
& +C\left(t\left\|f^{\prime}-g^{\prime}\right\|_{L^{\infty}\left(M_{-}, M_{+}\right)}+4 \sqrt{t} \sqrt{\left\|A^{\prime}-B^{\prime}\right\|_{L^{\infty}\left(M_{-}, M_{+}\right)}}\right),
\end{aligned}
$$


hence the mapping $S(t): L^{1}\left(\mathbb{R}^{d}\right) \times W^{1, \infty}\left(\left[M_{-}, M_{+}\right]\right) \times W^{1, \infty}\left(\left[M_{-}, M_{+}\right]\right) \rightarrow L^{1}\left(\mathbb{R}^{d}\right)$, $\left(u_{0}, f, A\right) \mapsto u(t, \cdot)$ is continuous as a mapping between Banach spaces if restricted to initial data $u_{0}$ in $U_{1}:=\left\{u_{0} \in L^{1}\left(\mathbb{R}^{d}\right): M_{-} \leq u_{0}(x) \leq M_{+}\right.$, a.e. $\left.x \in \mathbb{R}\right\} \subset L^{1}\left(\mathbb{R}^{d}\right)$ and $A$ satisfying $A^{\prime} \geq 0$.

\subsection{Random Entropy Solutions}

Existence and uniqueness for random initial data $u_{0}$ and random flux $f$ for $A \equiv 0$ for the Cauchy problem was proved in [28,34]; we now review these results. We remark that these results remain valid in bounded, axiparallel rectangles $D$ with periodicity conditions with respect to each variable.

Here we are interested in the case where the initial data $u_{0}$, the convective flux function $f$ and the diffusive flux function $A$ in (1.1) are uncertain, that is, random functions taking values in the Banach spaces $B V\left(\mathbb{R}^{d}\right) \cap L^{\infty}\left(\mathbb{R}^{d}\right)$ and $W^{1, \infty}(\mathbb{R} ; \mathbb{R})$ respectively.

To define these, we denote by $(\Omega, \mathcal{F}, \mathbb{P})$ a probability space. We consider spatially homogeneous random flux functions and diffusion operators $f$, $A$, i.e., strongly measurable maps $f: \Omega \rightarrow \operatorname{Lip}\left(\mathbb{R} ; \mathbb{R}^{d}\right), A: \Omega \rightarrow \operatorname{Lip}\left(\mathbb{R} ; \mathbb{R}^{d}\right)$, and random initial data $u_{0}$ being strongly measurable maps from $\Omega$ to the intersection of the Banach spaces $B V\left(\mathbb{R}^{d}\right)$ and $L^{\infty}\left(\mathbb{R}^{d}\right)$.

Definition 3.5. Random data for the scalar degenerate convection diffusion equation (1.1) is a random variable taking values in

$$
E_{1}=\left(B V\left(\mathbb{R}^{d}\right) \cap L^{\infty}\left(\mathbb{R}^{d}\right)\right) \times W^{1, \infty}\left(\mathbb{R} ; \mathbb{R}^{d}\right) \times W^{1, \infty}\left(\mathbb{R} ; \mathbb{R}^{d}\right) .
$$

The set $E_{1}$ is a Banach space which we equip with the norm

$$
\|(u, f, A)\|_{E_{1}}=\|u\|_{L^{1}\left(\mathbb{R}^{d}\right)}+\mathrm{TV}(u)+\|u\|_{L^{\infty}\left(\mathbb{R}^{d}\right)}+\|f\|_{W^{1, \infty}\left(\mathbb{R} ; \mathbb{R}^{d}\right)}+\|A\|_{W^{1, \infty}\left(\mathbb{R} ; \mathbb{R}^{d}\right)} .
$$

In particular, random data $\left(u_{0}, f, A\right)$ for the degenerate convection diffusion equation (1.1) is a strongly measurable map

$$
\left(u_{0}, f, A\right):(\Omega, \mathcal{F}) \longmapsto\left(E_{1}, \mathcal{B}\left(E_{1}\right)\right) .
$$

For the ensuing convergence analysis, we shall also require that for $\mathbb{P}$-a.e. $\omega$ it holds

$$
\begin{aligned}
& -\infty<M_{-} \leq u_{0}(\omega ; x) \leq M_{+}<\infty, \text { a.e. } x \in \mathbb{R}^{d}, \\
& \left|u_{0}(\omega ; \cdot)\right|_{B V\left(\mathbb{R}^{d}\right)} \leq C_{\mathrm{TV}}<\infty, \\
& \|f(\omega ; \cdot)\|_{W^{1, \infty}\left(\left[M_{-}, M_{+}\right]\right)} \leq C_{f}<\infty, \\
& A^{\prime}(\omega ; \cdot) \geq 0, \\
& \|A(\omega ; \cdot)\|_{W^{1, \infty}\left(\left[M_{-}, M_{+}\right]\right)} \leq C_{A}<\infty, \\
& \left|f\left(\omega ; u_{0}(\omega ; \cdot)\right)-\operatorname{grad} A\left(\omega ; u_{0}(\omega ; \cdot)\right)\right|_{B V\left(\mathbb{R}^{d}\right)} \leq C_{A, f}<\infty .
\end{aligned}
$$


We shall refer to a random flux $f$ which satisfies (3.16) as bounded random flux and similarly to $A$ satisfying (3.17)-(3.19) as bounded random diffusion operator. In addition, we shall assume

$$
\left\|u_{0}\right\|_{L^{r}\left(\Omega ; L^{p}\left(\mathbb{R}^{d}\right) \cap L^{1}\left(\mathbb{R}^{d}\right)\right)}<\infty
$$

for some $p, r \geq 1$. By (2.2), for random data with (3.14)-(3.20) the map

$$
\begin{gathered}
\Omega \ni \omega \\
\downarrow \\
\left(\left\|u_{0}(\omega ; \cdot)\right\|_{L^{1}\left(\mathbb{R}^{d}\right)}, \operatorname{TV}\left(u_{0}(\omega ; \cdot)\right),\left\|u_{0}(\omega ; \cdot)\right\|_{L^{\infty}\left(\mathbb{R}^{d}\right)},\|f\|_{W^{1, \infty}\left(\mathbb{R} ; \mathbb{R}^{d}\right)},\|A\|_{W^{1, \infty}\left(\mathbb{R} ; \mathbb{R}^{d}\right)}\right)
\end{gathered}
$$

is in $L^{r}\left(\Omega ; \mathbb{R}^{5}\right)$.

Then we are interested in random solutions of the random degenerate convection diffusion equation

$$
\left\{\begin{array}{l}
u_{t}(\omega ; x, t)+\operatorname{div}(f(\omega ; u(\omega ; x, t)))=\Delta A(\omega ; u(\omega ; x, t)), t>0, x \in \mathbb{R}^{d} \\
u(\omega ; x, 0)=u_{0}(\omega ; x), x \in \mathbb{R}^{d}
\end{array}\right.
$$

Definition 3.6. A random field $u: \Omega \ni \omega \rightarrow u(\omega ; x, t)$, i.e., a measurable mapping from $(\Omega, \mathcal{F})$ to $C\left([0, T] ; L^{1}\left(\mathbb{R}^{d}\right)\right)$, is called a random entropy solution of (3.1) with random initial data $u_{0}$, flux function $f$ and diffusive flux $A$ satisfying (3.13) and (3.14) - (3.21) for some $r \geq 1$, if it satisfies:

(i) Weak solution: for $\mathbb{P}$-a.e. $\omega \in \Omega, u(\omega ; \cdot, \cdot)$ satisfies

$$
\begin{array}{r}
\int_{0}^{\infty} \int_{\mathbb{R}^{d}}\left(u(\omega ; x, t) \varphi_{t}+(f(\omega ; u(\omega ; x, t))-\operatorname{grad} A(\omega ; u(\omega ; x, t))) \cdot \operatorname{grad} \varphi\right) d x d t \\
+\int_{\mathbb{R}^{d}} u_{0}(x, \omega) \varphi(x, 0) d x=0,
\end{array}
$$

for all test functions $\varphi \in C_{0}^{1}\left(\mathbb{R}^{d} \times[0, \infty)\right)$.

(ii) Entropy condition: For any pair consisting of a (deterministic) entropy $\eta$ and (stochastic) entropy flux $q(\omega ; \cdot)$ and $r(\omega ; \cdot)$ i.e., $\eta, q$ and $r$ are functions such that $\eta$ is convex and such that $q^{\prime}(\omega ; \cdot)=\eta^{\prime} f^{\prime}(\omega ; \cdot), r^{\prime}(\omega ; \cdot)=$ $\eta^{\prime} A^{\prime}(\omega ; \cdot)$ and for $\mathbb{P}$-a.s. $\omega \in \Omega$, u satisfies the following integral identity:

$$
\begin{array}{r}
\int_{0}^{\infty} \int_{\mathbb{R}^{d}}\left(\eta(u(\omega ; x, t)) \varphi_{t}+\operatorname{grad} q(\omega ; u(\omega ; x, t)) \cdot \operatorname{grad} \varphi+r(\omega ; u(\omega ; x, t)) \Delta \varphi\right) d x d t \\
+\int_{\mathbb{R}^{d}} \eta\left(u_{0}(\omega ; x)\right) \varphi(x, 0) d x \geq 0, \quad(3.23)
\end{array}
$$

for all test functions $0 \leq \varphi \in C_{0}^{1}\left(\mathbb{R}^{d} \times[0, \infty)\right)$. 
We state the following theorem regarding the random entropy solution of (3.22):

Theorem 3.7. Consider the degenerate convection diffusion equation (3.1) with random initial data $u_{0}$, flux function $f$ and random diffusion operator $A$, as in (3.13), and satisfying (3.14) - (3.20) for some $r \in[1, \infty]$.Then there exists a unique random entropy solution $u: \Omega \ni \omega \rightarrow C\left([0, T] ; L^{1}(\mathbb{R})\right)$ which is "pathwise", i.e., for $\mathbb{P}-$ a.s. $\omega \in \Omega$, described in terms of a nonlinear mapping $S(t)$ which depends only on the random flux and diffusion,

$$
u(\omega ; \cdot, t)=S(t)\left(u_{0}(\omega ; \cdot), f(\omega ; \cdot), A(\omega ; \cdot)\right), \quad t>0, \mathbb{P}-\text { a.e. } \omega \in \Omega
$$

such that for every $0 \leq t \leq T<\infty$ and for $s=1, p$;

$$
\begin{aligned}
\|u\|_{L^{r}\left(\Omega ; C\left([0, T] ; L^{s}\left(\mathbb{R}^{d}\right)\right)\right)} & \leq\left\|u_{0}\right\|_{L^{r}\left(\Omega ; L^{s}\left(\mathbb{R}^{d}\right)\right)}, \\
\left\|S(t)\left(u_{0}, f, A\right)(\omega)\right\|_{\left(L^{1} \cap L^{\infty}\right)\left(\mathbb{R}^{d}\right)} & \leq\left\|u_{0}(\omega ; \cdot)\right\|_{\left(L^{1} \cap L^{\infty}\right)\left(\mathbb{R}^{d}\right)} .
\end{aligned}
$$

Moreover, we have $\mathbb{P}$-a.s.

$$
\begin{gathered}
\left|S(t)\left(u_{0}, f, A\right)(\omega)\right|_{B V\left(\mathbb{R}^{d}\right)} \leq\left|u_{0}(\omega ; \cdot)\right|_{B V\left(\mathbb{R}^{d}\right)}, \\
|f(\omega ; u(\omega ; \cdot, t))-\operatorname{grad} A(\omega ; u(\omega ; \cdot, t))|_{B V\left(\mathbb{R}^{d}\right)} \\
\leq\left|f\left(\omega ; u_{0}(\omega ; \cdot)\right)-\operatorname{grad} A\left(\omega ; u_{0}(\omega ; \cdot)\right)\right|_{B V\left(\mathbb{R}^{d}\right)}, \\
\left\|u\left(\omega ; \cdot, t_{1}\right)-u\left(\omega ; \cdot, t_{2}\right)\right\|_{L^{1}\left(\mathbb{R}^{d}\right)} \\
\leq\left|f\left(\omega ; u_{0}(\omega ; \cdot)\right)-\operatorname{grad} A\left(\omega ; u_{0}(\omega ; \cdot)\right)\right|_{B V\left(\mathbb{R}^{d}\right)}\left|t_{1}-t_{2}\right| . \\
\text { and, with } \bar{M}:=\max \left\{\left|M_{-}\right|,\left|M_{+}\right|\right\} \text {for } M_{-}, M_{+} \text {as in }(3.14), \\
\sup _{0 \leq t \leq T}\|u(\omega ; \cdot, t)\|_{L^{\infty}\left(\mathbb{R}^{d}\right)} \leq \bar{M} \quad \mathbb{P}-\text { a.s. } \omega \in \Omega .
\end{gathered}
$$

Proof. For $\omega \in \Omega$, we define, motivated by Theorem 3.1, for $\mathbb{P}$-a.e. $\omega \in \Omega$ a random function $u(\omega ; t, x)$ by

$$
u(\omega ; \cdot)=S(t)\left(u_{0}, f, A\right)(\omega) .
$$

By the properties of the solution mapping $(S(t))_{t \geq 0}$, see Theorem 3.1, the random field defined in (3.30) is well defined; for $\mathbb{P}$-a.e. $\omega \in \Omega, u(\omega ; \cdot)$ is a weak entropy solution of the degenerate diffusion equation (3.1). Moreover, we obtain from Theorem 3.1 that $\mathbb{P}$-a.s. all bounds (3.25)-(3.28) hold, with assumption (3.14) also (3.29). The measurability of the mapping $\Omega \ni \omega \mapsto u(\omega ; \cdot, t) \in L^{1}(\mathbb{R})$, $0 \leq t \leq T$ follows from Lemma 2.1, (3.11) and the assumption that the mapping $\Omega \ni \omega \mapsto\left(u_{0}, f, A\right)(\omega) \in E_{1}$ is a random variable. Finally, (3.24) follows from (3.20) together with (3.5) in Theorem 3.1.

Theorem 3.7 generalizes the existence of random entropy solutions for random initial data from [29] and random convective flux function [28]. 
Remark 3.8. All existence and continuous dependence results stated so far are formulated for the deterministic Cauchy problem (3.1). By the 'usual arguments', verbatim the same results will also hold for solutions defined in a bounded, axiparallel domain $D \subset \mathbb{R}^{d}$, provided that periodic boundary conditions in each space coordinate are enforced on the weak solutions.

\section{Numerical approximation of random degenerate convection diffusion equation}

We wish to compute various quantities of interest, such as the expectation of the solution $u$ to the random degenerate diffusion equation (3.22). We choose to split the approximation into two steps: On one hand, we need to approximate in the stochastic domain $\omega \in \Omega$ and on the other hand, since in general exact solutions to (1.1) are not available, we need an approximation in the physical domain $(x, t) \in \Pi_{T}$. In this paper, we will consider a Multilevel Monte Carlo Finite Difference Method (MLMC-FDM), that is, a combination of the multilevel Monte Carlo method with a deterministic finite difference discretization. We will briefly review the two methods and mention some relevant results in the following sections.

\subsection{Monte Carlo method}

To "discretize" the stochastic domain, we will use the Monte Carlo method as described in Section 2.2. We again assume that $\left(u_{0}(\omega ; \cdot), f(\omega ; \cdot), A(\omega ; \cdot)\right) \in E_{1}$ and that satisfy in addition (3.14)-(3.19) and (3.20) for some $r, p \in[1, \infty)$ to be specified later. We shall be interested in the statistical estimation of the first moment of $u$, i.e. $\mathbb{E}[u]$. The Monte Carlo $(M C)$ approximation of $\mathbb{E}[u]$ is defined as follows: given $M$ independent, identically distributed samples $\left(\widehat{u}_{0}^{i}, \widehat{f}^{i}, \widehat{A}^{i}\right), i=1, \ldots, M$, of initial data, flux function and diffusion, the MC estimate of $\mathbb{E}[u(\cdot, t ; \cdot)]$ at time $t$ is given by

$$
E_{M}[u(\cdot, t)]:=\frac{1}{M} \sum_{i=1}^{M} \widehat{u}^{i}(\cdot, t)
$$

where $\widehat{u}^{i}(\cdot, t)$ denote the $M$ unique entropy solutions of the $M$ Cauchy problems (1.1) with initial data $\widehat{u}_{0}^{i}$, flux function $\widehat{f}^{i}$ and diffusion operator $\widehat{A}^{i}$. Since

$$
\widehat{u}^{i}(\cdot, t)=S(t)\left(\widehat{u}_{0}^{i}, \widehat{f}^{i}, \widehat{A}^{i}\right),
$$

we have for every $M$ and for every $0<t<\infty$, by (3.5) (for $p=1$ ) or the entropy $(p>1)$ condition (3.23) (cf. Remark 3.2),

$$
\begin{aligned}
\left\|E_{M}[u(\cdot, t ; \omega)]\right\|_{L^{p}\left(\mathbb{R}^{d}\right)} & =\left\|\frac{1}{M} \sum_{i=1}^{M}, S(t)\left(\widehat{u}_{0}^{i}, \widehat{f}^{i}, \widehat{A}^{i}\right)(\omega)\right\|_{L^{p}\left(\mathbb{R}^{d}\right)} \\
& \leq \frac{1}{M} \sum_{i=1}^{M}\left\|S(t)\left(\widehat{u}_{0}^{i}, \widehat{f}^{i}, \widehat{A}^{i}\right)(\omega)\right\|_{L^{p}\left(\mathbb{R}^{d}\right)}
\end{aligned}
$$


14 Koley, Risebro, Schwab, Weber

$$
\leq \frac{1}{M} \sum_{i=1}^{M}\left\|\widehat{u}_{0}^{i}(\cdot ; \omega)\right\|_{L^{p}\left(\mathbb{R}^{d}\right)} .
$$

Using this inequality, the i.i.d. property of the samples $\left\{\left(\widehat{u}_{0}^{i}, \widehat{f}^{i}, \widehat{A}^{i}\right)\right\}_{i=1}^{M}$, we obtain the bound

$$
\begin{aligned}
\mathbb{E}\left[\left\|E_{M}[u(\cdot, t)]\right\|_{L^{p}\left(\mathbb{R}^{d}\right)}\right] & \leq \mathbb{E}\left[\frac{1}{M} \sum_{i=1}^{M}\left\|\widehat{u}_{0}^{i}(\cdot ; \omega)\right\|_{L^{p}\left(\mathbb{R}^{d}\right)}\right] \\
& =\mathbb{E}\left[\left\|u_{0}\right\|_{L^{p}\left(\mathbb{R}^{d}\right)}\right]=\left\|u_{0}\right\|_{L^{1}\left(\Omega ; L^{p}\left(\mathbb{R}^{d}\right)\right)}<\infty .
\end{aligned}
$$

Theorem 4.1. Assume that in (3.22) the random variable $\left(u_{0}, f, A\right)(\omega)$ as in (3.13) satisfies (3.14) - (3.19) and that $A^{\prime}(\omega ; \cdot) \geq 0$, for $\mathbb{P}$-a.s. $\omega \in \Omega$ and, for some $r \in] 1, \infty[, p \geq 1$,

$$
u_{0} \in L^{r}\left(\Omega ; L^{p}\left(\mathbb{R}^{d}\right)\right) .
$$

Then the Monte Carlo estimates $E_{M}[u(\cdot, t)]$ in $(4.1)$ converge in $L^{q}\left(\Omega ; L^{p}\left(\mathbb{R}^{d}\right)\right)$ for $q:=\min \{2, p, r\}>1$ as $M \rightarrow \infty$, to $\mathcal{M}^{1}(u(\cdot, t))=\mathbb{E}[u(\cdot, t)]$. In addition, for any $M \in \mathbb{N}, 0<t<\infty$, we have the error bound

$$
\left\|\mathbb{E}[u(\cdot, t)]-E_{M}[u(\cdot, t)]\right\|_{L^{q}\left(\Omega ; L^{p}\left(\mathbb{R}^{d}\right)\right)}^{q} \leq C M^{1-q}\left\|u_{0}\right\|_{L^{q}\left(\Omega ; L^{p}\left(\mathbb{R}^{d}\right)\right)}^{q} .
$$

Proof. By the linearity of expectation, we have

$$
\begin{aligned}
\left\|\mathbb{E}[u(\cdot, t)]-E_{M}[u(\cdot, t)]\right\|_{L^{q}\left(\Omega ; L^{p}\left(\mathbb{R}^{d}\right)\right)}^{q} & =\mathbb{E}\left[\left\|\mathbb{E}[u(\cdot, t)]-E_{M}[u(\cdot, t)]\right\|_{L^{p}\left(\mathbb{R}^{d}\right)}^{q}\right] \\
& =\mathbb{E}\left[\left\|\frac{1}{M} \sum_{i=1}^{M}\left(\mathbb{E}[u(\cdot, t)]-\widehat{u}^{i}(\cdot, t)\right)\right\|_{L^{p}\left(\mathbb{R}^{d}\right)}^{q}\right] .
\end{aligned}
$$

It follows from Remark 2.3 (iii), that the Banach space $E=L^{p}\left(\mathbb{R}^{d}\right)$ is of type $q=\min \{2, p, r\}>1$. Hence we can apply Corollary 2.5 to the iid random variables $Y_{i}:=\mathbb{E}[u(\cdot, t)]-\widehat{u}^{i}(\cdot, t)$ which have zero mean in the last term to estimate

$$
\begin{aligned}
\mathbb{E}\left[\left\|\frac{1}{M} \sum_{i=1}^{M}\left(\mathbb{E}[u(\cdot, t)]-\widehat{u}^{i}(\cdot, t)\right)\right\|_{L^{p}\left(\mathbb{R}^{d}\right)}^{q}\right] & :=\mathbb{E}\left[\left\|\frac{1}{M} \sum_{i=1}^{M} Y_{i}\right\|_{L^{p}\left(\mathbb{R}^{d}\right)}^{q}\right] \\
& \leq \frac{\left(2 C_{t}\right)^{q}}{M^{q-1}} \mathbb{E}\left[\left\|Y_{1}\right\|_{L^{p}\left(\mathbb{R}^{d}\right)}^{q}\right] \\
& =\frac{C}{M^{q-1}} \mathbb{E}\left[\|\mathbb{E}[u(\cdot, t)]-u(\cdot, t)\|_{L^{p}\left(\mathbb{R}^{d}\right)}^{q}\right] \\
& \leq \frac{C}{M^{q-1}} \mathbb{E}\left[\|u(\cdot, t)\|_{L^{p}\left(\mathbb{R}^{d}\right)}^{q}\right] .
\end{aligned}
$$


Using the entropy condition (3.23) (cf. Remark 3.2), the last expression can be bounded by

$$
\frac{C}{M^{q-1}} \mathbb{E}\left[\|u(\cdot, t)\|_{L^{p}\left(\mathbb{R}^{d}\right)}^{q}\right] \leq \frac{C}{M^{q-1}} \mathbb{E}\left[\left\|u_{0}\right\|_{L^{p}\left(\mathbb{R}^{d}\right)}^{q}\right],
$$

and the claim follows.

\subsection{Finite Difference Methods for degenerate convection diffusion equations}

So far, we considered the MCM under the assumption that the entropy solutions $\widehat{u}^{i}(x, t ; \omega)=S(t)\left(\widehat{u}_{0}^{i}, \widehat{f}^{i}, \widehat{A}^{i}\right)(\omega)$ for the Cauchy problem (1.1) with the data samples $\left(\hat{u}_{0}^{i}, \widehat{f}^{i}, \widehat{A}^{i}\right)$ are available exactly. In practice, however, we must use numerical approximations of $S(t)\left(\hat{u}_{0}^{i}, \widehat{f}^{i}, \widehat{A}^{i}\right)$. We next present a family of convergent discretization schemes, with corresponding stability and consistency bounds.

\subsubsection{Definition, Stability and Consistency of the Scheme}

The presentation will, from now on, be restricted to the one-dimensional case, that is, we consider

$$
\left\{\begin{array}{l}
u_{t}+f(u)_{x}=A(u)_{x x}, \quad t>0, x \in D \subset \mathbb{R}, \\
u(x, 0)=u_{0}(x) .
\end{array}\right.
$$

We thus assume that $D \subset \mathbb{R}$ is a bounded interval in $\mathbb{R}$ and we shall consider $D$ periodic solutions $u$. We shall examine the class of fully discrete monotone difference schemes for which Karlsen, Risebro and Storrøsten obtained a convergence in $L^{1}$ rate of $\Delta x^{1 / 3}$, where $\Delta x$ is the discretization parameter, in [20]. These schemes are easily generalized to several space dimensions, but rigorous results regarding convergence rates are much worse. To date, the best convergence rate in $L^{1}\left(\mathbb{R}^{d}\right)$ for a fully discrete, implicit in time scheme is $\Delta x^{2 /(19+d)}$, see [21].

For $\Delta x, \Delta t>0$, we discretize the space-time cylinder $\Pi_{T}=D \times[0, T]$ by a grid with cells

$$
I_{j}^{n}=\left[x_{j-1 / 2}, x_{j+1 / 2}\right) \times\left(t_{n-1}, t_{n}\right], \quad n \geq 0, j \in \mathcal{N}_{x},
$$

where $x_{j \pm 1 / 2}=(j \pm 1 / 2) \Delta x, j \in \mathcal{N}_{x}$, and $t_{n}=n \Delta t, n \in \mathbb{N} . \mathcal{N}_{x}:=\left\{N_{1}, N_{1}+\right.$ $\left.1, \ldots, N_{2}-1, N_{2}\right\}$, where $N_{1}, N_{2} \in \mathbb{Z}$ are such that $|D| /\left|N_{2}-N_{1}\right|=\Delta x$ and $x_{N_{1}-1 / 2}=\inf _{x} D$ and $x_{N_{2}+1 / 2}=\sup _{x} D$. We define cell averages of the initial data via

$$
u_{j}^{0}=\frac{1}{\Delta x} \int_{I_{j}^{0}} u_{0}(x) d x, \quad j \in \mathcal{N}_{x}
$$

Then we consider the following implicit scheme

$$
D_{-}^{t} u_{j}^{n}+D_{-} F\left(u_{j}^{n}, u_{j+1}^{n}\right)=D_{-} D_{+} A\left(u_{j}^{n}\right), \quad n \geq 1, j \in \mathcal{N}_{x},
$$


and the explicit scheme,

$$
D_{+}^{t} u_{j}^{n}+D_{-} F\left(u_{j}^{n}, u_{j+1}^{n}\right)=D_{-} D_{+} A\left(u_{j}^{n}\right), \quad n \geq 0, j \in \mathcal{N}_{x},
$$

where we have denoted for a quantity $\left\{\sigma_{j}^{n}\right\}_{j \in \mathcal{N}_{x}, n \in \mathbb{N}}$,

$$
D_{ \pm}^{t} \sigma_{j}^{n}= \pm \frac{1}{\Delta t}\left(\sigma_{j}^{n \pm 1}-\sigma_{j}^{n}\right), \quad D_{ \pm} \sigma_{j}^{n}= \pm \frac{1}{\Delta x}\left(\sigma_{j \pm 1}^{n}-\sigma_{j}^{n}\right) .
$$

We then define the piecewise constant approximation to (4.3) by

$$
u_{\Delta}(x, t)=u_{j}^{n}, \quad(x, t) \in I_{j}^{n},
$$

where $u_{j}^{n}$ is defined by either (4.5) or (4.6). The numerical flux $F \in C^{1}\left(\mathbb{R}^{2}\right)$ is chosen such that it is consistent with $f$, that is, $F(u, u)=f(u)$ for all $u \in \mathbb{R}$, and monotone, which means

$$
\frac{\partial}{\partial u} F(u, v) \geq 0 \quad \text { and } \quad \frac{\partial}{\partial v} F(u, v) \leq 0 .
$$

In order to obtain convergence rates, it is furthermore necessary to choose $F$ Lipschitz continuous and such that it can be written

$$
F(u, v)=F_{1}(u)+F_{2}(v), \quad F_{1}^{\prime}(u)+F_{2}^{\prime}(u)=f^{\prime}(u),
$$

see [20]. Examples of monotone numerical fluxes satisfying (4.8) are the EngquistOsher flux as well as the Lax-Friedrichs and the upwind flux. In order to show convergence of the explicit scheme, the following CFL-condition is needed [11],

$$
\Delta t \leq C \Delta x^{2}
$$

and in order to show a convergence rate, one even needs

$$
\Delta t \leq C \Delta x^{8 / 3},
$$

see [20]. Whether this restrictive CFL-condition is sharp in order to prove a convergence rate is not known. Naturally, no CFL-condition is needed to ensure stability of the implicit scheme, [13]. In order to obtain à priori estimates for the explicit scheme, the numerical flux function $F$ and the diffusion operator $A$ have to satisfy the following condition

$$
\frac{\Delta t}{\Delta x}\left(F_{1}^{\prime}(z)-F_{2}^{\prime}(z)\right)+2 \frac{\Delta t}{\Delta x^{2}} A^{\prime}(w) \leq 1, \quad \text { for all } z \text { and } w,
$$

see [11]. Then we have the following stability and convergence results for the schemes (4.5) and (4.6), $[11,9,20]$ (which also hold for the bounded domain $D$ replaced by R.)

Theorem 4.2. Let $u_{0} \in B V(D) \cap L^{1}(D), M_{-} \leq u_{0} \leq M_{+}, f, A \in \operatorname{Lip}\left(\left[M_{-}, M_{+}\right]\right)$, $A^{\prime} \geq 0$ and $u_{0} \in \mathcal{A}(f, A)$, where $\mathcal{A}(f, A)$ is defined in (3.2). Let $F$ be a monotone numerical flux function consistent with $f$, satisfying (4.8). Denote by $u_{\Delta}(x, t)$ the piecewise constant function defined in (4.7), where $u_{j}^{n}$ are computed by either the explicit scheme (4.6) or the implicit scheme (4.5). Assume for the explicit scheme in addition that $\Delta t$ satisfies (4.9) and that (4.11) holds. Then we have 
i) The approximations $u_{\Delta}$ converge, as the discretization parameters $(\Delta x, \Delta t) \rightarrow 0$ subject to the CFL condition (4.9) and (4.11), to the unique entropy solution of (4.3). Moreover they satisfy

$$
\begin{aligned}
\left\|u_{\Delta}(\cdot, t)\right\|_{L^{1}(D)} & \leq\left\|u_{0}\right\|_{L^{1}(D)}, \\
\left\|u_{\Delta}(\cdot, t)\right\|_{L^{\infty}(D)} & \leq\left\|u_{0}\right\|_{L^{\infty}(D)}, \\
\left|u_{\Delta}(\cdot, t)\right|_{B V(D)} & \leq\left|u_{0}\right|_{B V(D)}, \\
\sup _{j}\left|F\left(u_{j}^{n}, u_{j+1}^{n}\right)-D_{+} A\left(u_{j}^{n}\right)\right| & \leq \sup _{j}\left|F\left(u_{j}^{0}, u_{j+1}^{0}\right)-D_{+} A\left(u_{j}^{0}\right)\right|, \\
\sum_{j}\left|D_{-} F\left(u_{j}^{n}, u_{j+1}^{n}\right)-D_{-} D_{+} A\left(u_{j}^{n}\right)\right| & \leq \sum_{j}\left|D_{-} F\left(u_{j}^{0}, u_{j+1}^{0}\right)-D_{-} D_{+} A\left(u_{j}^{0}\right)\right| .
\end{aligned}
$$

Furthermore, $u_{\Delta}$ is $L^{1}(D)$-Lipschitz continuous in time, viz., for any $t_{n}$, $t_{m}>0$,

$$
\left\|u_{\Delta}\left(\cdot, t_{n}\right)-u_{\Delta}\left(\cdot, t_{m}\right)\right\|_{L^{1}(D)} \leq\left|f\left(u_{0}\right)-A\left(u_{0}\right)\right|_{B V(D)}\left|t_{n}-t_{m}\right| .
$$

ii) If for the explicit scheme in addition (4.10) holds, the approximations $u_{\Delta}$ converge at the rate $1 / 3$ to the entropy solution $u$ of (4.3):

$$
\left\|u_{\Delta}\left(\cdot, t_{n}\right)-u\left(\cdot, t_{n}\right)\right\|_{L^{1}(D)} \leq\left\|u_{\Delta}(\cdot, 0)-u_{0}\right\|_{L^{1}(D)}+C_{T} \Delta x^{1 / 3}
$$

where the constant $C_{T}$ takes the form

$C(1+T)\left(\left(1+\|f\|_{\text {Lip }}\right)\left|u_{0}\right|_{B V(D)}+\left\|A\left(u_{0}\right)_{x}\right\|_{L^{1}(D)}+\left|f\left(u_{0}\right)-A\left(u_{0}\right)_{x}\right|_{B V(D)}\right)$, with $C$ independent of $u_{0}, f$ and $A$.

Point i) was proved in [11, Thm. 3.9, Cor. 3.10] for the explicit scheme and [9, Thm. 3.9, Lem. 3.3, 3.4, 3.5] for the implicit scheme, ii) in [20].

Remark 4.3. Combining (4.12), (3.4) respectively, with (4.13), we can obtain a (possibly not optimal) estimate for the rate of convergence of the scheme in $L^{p}(D)$ for $1 \leq p<\infty$ using Hölder's inequality:

$$
\begin{aligned}
\left\|u_{\Delta}\left(\cdot, t_{n}\right)-u\left(\cdot, t_{n}\right)\right\|_{L^{p}(D)} & \leq\left\|u_{\Delta}\left(\cdot, t_{n}\right)-u\left(\cdot, t_{n}\right)\right\|_{L^{1}(D)}^{1 / p}\left\|u_{\Delta}\left(\cdot, t_{n}\right)-u\left(\cdot, t_{n}\right)\right\|_{L^{\infty}(D)}^{1-1 / p} \\
& \leq C\left(\left\|u_{\Delta}(\cdot, 0)-u_{0}\right\|_{L^{1}(D)}^{1 / p}+C_{T}^{1 / p} \Delta x^{1 /(3 p)}\right)
\end{aligned}
$$

where the constant $C$ is dependent on the $L^{\infty}$-norm of $u_{0}$.

\subsubsection{Work Bounds}

For the purpose of analyzing the efficiency of the MC- and MLMC-method, it is important to have an estimate on the computational work used to compute one approximation of the solution by the deterministic FD-schemes and how it increases with respect to mesh refinement. By (computational) work or cost of an algorithm, 
we mean the number of floating point operations performed during the execution of the algorithm. We assume that this is proportional to the run time of the algorithm. Because we deal with bounded domains in the actual computations, the number of grid cells in one dimension scales as $1 / \Delta x$.

Work estimate for the explicit scheme (4.6). For the explicit scheme, the number of operations per time step scales linearly with the number of cells in the spatial domain $D$, which in turn scales as $\Delta x^{-1}$ (we assume the computational domain is bounded). Hence the work can be bounded as $W_{\Delta}^{\exp } \leq C \Delta t^{-1} \Delta x^{-1}$. Taking the CFL-condition (4.10) into account, we obtain the (likely pessimistic) work bound

$$
W_{\Delta}^{\mathrm{ex}}=\mathcal{O}\left(\Delta x^{-11 / 3}\right) \text {. }
$$

Work estimate for the implicit scheme (4.5). In the implicit scheme we have to solve the nonlinear equation (4.5) for $\underline{u}^{n+1}:=\left(\ldots, u_{j-1}^{n+1}, u_{j}^{n+1}, u_{j+1}^{n+1}, \ldots\right)$ in each timestep. Since solving this equation exactly is either impossible or computationally very expensive, we prefer to solve it only approximately by an iterative method. We consider here the case that this method is the Newton iteration, which we iterate until the residual is of order $\Delta x \Delta t$ (this is possible since the mapping $\underline{u}^{n} \rightarrow \underline{u}^{n+1}=$ : $\Psi\left(\underline{u}^{n}\right)$ defined by (4.5) is a contraction for sufficiently small $\Delta t$ and CFL constant.

In general the Lipschitz constant should scale as $1 / \Delta x$, so a small value of $\Delta t$ alone is not sufficient for the contraction property to hold. For details, we refer to [9]. The additional error introduced by finite termination of the iterative nonlinear system solver will not increase the overall error: denoting by $\underline{u}^{n,(0)}$ the approximation at time $t=t_{n}$ obtained by solving (4.5) exactly in each time step, $\underline{u}^{n,(j)}$ the approximation obtained by solving (4.5) approximately via Newton iteration in the first $j$ timesteps and afterwards exactly (so that $\underline{u}^{n,(n)}:=u_{\Delta}\left(\cdot, t_{n}\right.$ ) is the approximation obtained by using Newton's method in each timestep), we have

$$
\begin{aligned}
\left\|u_{\Delta}\left(\cdot, t_{n}\right)-u\left(\cdot, t_{n}\right)\right\|_{L^{1}(D)} & =\left\|\underline{u}^{n,(n)}-u\left(\cdot, t_{n}\right)\right\|_{L^{1}(D)} \\
& =\left\|\sum_{m=0}^{n-1}\left(\underline{u}^{n,(m+1)}-\underline{u}^{n,(m)}\right)+\underline{u}^{n,(0)}-u\left(\cdot, t_{n}\right)\right\|_{L^{1}(D)} \\
& \leq \sum_{m=0}^{n-1}\left\|\underline{u}^{n,(m+1)}-\underline{u}^{n,(m)}\right\|_{L^{1}(D)}+\left\|\underline{u}^{n,(0)}-u\left(\cdot, t_{n}\right)\right\|_{L^{1}(D)} \\
& \leq \sum_{m=0}^{n-1}\left\|\underline{u}^{m+1,(m+1)}-\underline{u}^{m+1,(m)}\right\|_{L^{1}(D)}+C_{T} \Delta x^{1 / 3} \\
& \leq n \Delta x \Delta t+C_{T} \Delta x^{1 / 3} \\
& =t_{n} \Delta x+C_{T} \Delta x^{1 / 3} \leq \widetilde{C}_{T} \Delta x^{1 / 3},
\end{aligned}
$$

where we have used the $L^{1}$-contraction property of the scheme for the third last inequality. If the starting value for the Newton iteration is chosen such that it is in a sufficiently small neighborhood of the fixpoint, the convergence order of the Newton method is locally quadratic. In order to achieve an error of less than $C \Delta x \Delta t$ 
in one timestep by solving the nonlinear system only approximately, it suffices to perform $\mathcal{O}\left(\log \left(\Delta x^{-1} \Delta t^{-1}\right)\right)$ many Newton iterations. If we take $\Delta t=\theta \Delta x$ for some constant $\theta>0$, these are altogether $\mathcal{O}\left(\log \left(\Delta x^{-2}\right)\right)=\mathcal{O}\left(\log \left(\Delta x^{-1}\right)\right)$ Newton steps. In each step of the Newton iteration, we invert and multiply a tridiagonal matrix of size $\mathcal{O}\left(\Delta x^{-2}\right)$ with a vector of length $\mathcal{O}\left(\Delta x^{-1}\right)$ and subtract it from another vector of length $\mathcal{O}\left(\Delta x^{-1}\right)$. The tridiagonal matrix can be inverted in $\mathcal{O}\left(\Delta x^{-1}\right)$ operations using the Thomas algorithm (in case of periodic boundary conditions we use the Sherman-Morrison formula). Hence the total number of floating point operations which are necessary for one Newton step is $\mathcal{O}\left(\Delta x^{-1}\right)$. It follows that the work done in one timestep is of order $\mathcal{O}\left(\log \left(\Delta x^{-1}\right) \Delta x^{-1}\right)$. As there are altogether $n=T / \Delta t$ timesteps, and since we can choose the timestep of order $\Delta t=\theta \Delta x$, we obtain the following bound on the total work for one execution of the implicit scheme,

$$
W_{\Delta}^{\mathrm{im}}=\mathcal{O}\left(\Delta x^{-2} \log \left(\Delta x^{-1}\right)\right) .
$$

\subsubsection{Application to random data}

In the Monte Carlo Finite Difference Methods (MC-FDMs), we combine MC sampling of the random initial data with the FDMs (4.5) and (4.6). In the convergence analysis of these schemes, we shall require the application of the FDMs (4.5) and (4.6) to random initial data, flux function and diffusion operator $\left(u_{0}, f, A\right) \in$ $L^{p}\left(\Omega ; E_{1}\right)$ for some $1 \leq p \leq \infty$. Given a draw $\left(u_{0}(\omega ; \cdot), f(\omega ; \cdot), A(\omega ; \cdot)\right)$ of $\left(u_{0}, f, A\right)$, the FDMs (4.4) with (4.6) or (4.5) define families $u_{\Delta}(\omega ; x, t)$ of grid functions. We have the following stability and consistency estimates, which hold uniformly, i.e. $\mathbb{P}$-a.s. with respect to $\omega$.

Proposition 4.4. Consider the FDMs (4.4)-(4.6), (4.5) for the approximation of the entropy solution corresponding to the draw $\left(u_{0}, f, A\right)(\omega)$ of the random data.

Then, the random grid functions $\Omega \ni \omega \longmapsto u_{\Delta}(\omega ; x, t)$ defined by (4.7) satisfy, for every $0<\bar{t}<\infty, 0<\Delta x<1$, and every $r \geq 1$ the stability bounds:

$$
\begin{gathered}
\left\|u_{\Delta}(\cdot ; \cdot, \bar{t})\right\|_{L^{r}\left(\Omega ; L^{\infty}(D)\right)} \leq\left\|u_{0}\right\|_{L^{r}\left(\Omega ; L^{\infty}(D)\right)}, \\
\left\|u_{\Delta}(\cdot ; \cdot \bar{t})\right\|_{L^{r}\left(\Omega ; L^{1}(D)\right)} \leq\left\|u_{0}\right\|_{L^{r}\left(\Omega ; L^{1}(D)\right)} .
\end{gathered}
$$

We also have the consistency bound: there exists a constant $C>0$ independent of $\bar{t}$ and of $\Delta$, such that

$$
\begin{aligned}
\| u(\cdot ; \cdot, \bar{t}) & -u_{\Delta}(\cdot ; \cdot \bar{t})\left\|_{L^{r}\left(\Omega ; L^{1}(D)\right)} \leq\right\| u_{0}-u_{\Delta}(\cdot ; \cdot, 0) \|_{L^{r}\left(\Omega ; L^{1}(D)\right)} \\
& +C(1+\bar{t}) \Delta x^{1 / 3}\left\{\left\|\left(1+\|f(\omega ; \cdot)\|_{\text {Lip }}\right)\left|\left(u_{0}\right)(\omega)\right|_{B V(D)}\right\|_{L^{r}(\Omega)}\right. \\
& \left.+\left\|A\left(u_{0}\right)_{x}\right\|_{L^{r}\left(\Omega ; L^{1}(D)\right)}+\left\|\left|\left(f\left(u_{0}\right)-A\left(u_{0}\right)_{x}\right)(\omega)\right|_{B V(D)}\right\|_{L^{r}(\Omega)}\right\} .
\end{aligned}
$$

Remark 4.5. Reasoning as in Remark 4.3, under the assumptions (3.14) - (3.19), (4.15) becomes 


$$
\begin{aligned}
& \left\|u(\cdot ; \cdot, \bar{t})-u_{\Delta}(\cdot ; \cdot, \bar{t})\right\|_{L^{r}\left(\Omega ; L^{p}(D)\right)} \leq C \bar{M}^{1-1 / p}\left\|u_{0}-u_{\Delta}(\cdot ; \cdot, 0)\right\|_{L^{r}\left(\Omega ; L^{1}(D)\right)}^{1 / p} \\
& +C \bar{M}^{1-1 / p}\left((1+\bar{t}) \Delta x^{1 / 3}\left\{\left(1+C_{f}+C_{A}\right) C_{\mathrm{TV}}+C_{A, f}\right\}\right)^{1 / p}
\end{aligned}
$$

where $\bar{M}=\max \left\{\left|M_{-}\right|,\left|M_{+}\right|\right\}$(c.f. (3.14)).

\subsection{MC-FDM Scheme}

We next define and analyze the MC-FDM scheme. It is based on the straightforward idea of generating, possibly in parallel, independent samples of the random initial data and then, for each sample of the random initial data, flux function and diffusion operator, to perform one FD simulation. The error of this procedure is bounded by two contributions: a (statistical) sampling error and a (deterministic) discretization error. We express the asymptotic efficiency of this approach (in terms of overall error versus work). It will be seen that the efficiency of the MC-FDM is, in general, inferior to that of the deterministic schemes (4.6) and (4.5). The present analysis will constitute a key technical tool in our subsequent development and analysis of the multilevel MC-FDM ("MLMC-FDM" for short) which does not suffer from this drawback.

\subsubsection{Definition of the MC-FDM Scheme}

We consider once more the initial value problem (3.22) with random data $\left(u_{0}, f, A\right)$ satisfying (3.14) - (3.19) and (3.20) for sufficiently large $r \in \mathbb{R}$ (to be specified in the convergence analysis). The MC-FDM scheme for the MC estimation of the mean of the random entropy solutions then consists in the following:

Definition 4.6. (MC-FDM Scheme) Given $M \in \mathbb{N}$, generate $M$ i.i.d. samples $\left\{\left(\widehat{u}_{0}^{i}, \widehat{f}^{i}, \widehat{A}^{i}\right)\right\}_{i=1}^{M}$. Let $\left\{\widehat{u}^{i}(\cdot, t)\right\}_{i=1}^{M}$ denote the unique entropy solutions of the degenerate convection diffusion equations (1.1) for these data samples, i.e.

$$
\widehat{u}^{i}(\cdot, t)=S(t)\left(\widehat{u}_{0}^{i}, \widehat{f}^{i}, \widehat{A}^{i}\right), \quad i=1, \ldots, M .
$$

Then the MC-FDM approximations of $\mathcal{M}^{k}(u(\cdot, t))$ are defined as statistical estimates from the ensemble

$$
\left\{\widehat{u}_{\Delta}^{i}(\cdot, t)\right\}_{i=1}^{M}
$$

obtained from the FD approximations by (4.6) or (4.5) of (1.1) with data samples $\left\{\left(\widehat{u}_{0}^{i}, \widehat{f}^{i}, \widehat{A}^{i}\right)\right\}_{i=1}^{M}$ : Specifically, the first moment of the random solution $u(\omega ; \cdot, t)$ at time $t>0$, is estimated as

$$
\mathcal{M}^{1}(u(\cdot, t)) \approx E_{M}\left[u_{\Delta}(\cdot, t)\right]:=\frac{1}{M} \sum_{i=1}^{M} \widehat{u}_{\Delta}^{i}(\cdot, t) .
$$




\subsubsection{Convergence Analysis of MC-FDM}

We next address the convergence of $E_{M}\left[u_{\Delta}\right]$ to the mean $\mathbb{E}[u]$. We combine Theorem 4.1 for the convergence of the Monte Carlo method with the error estimate Proposition 4.4 for the finite difference method to obtain,

Theorem 4.7 (MC-FD Error bound). Assume that

$$
u_{0} \in L^{r}\left(\Omega ; L^{1}(D) \cap B V(D)\right)
$$

for some $r \in] 1, \infty]$ and that (3.14) - (3.19) hold. In addition, assume that $D$ is a bounded, axiparallel rectangle and periodic boundary conditions for $u$. Then the $M C$ estimate $E_{M}\left[u_{\Delta}(\cdot, t)\right]$ defined in (4.16) as in Definition 4.6 satisfies, for every $M$, and for $q=\min \{2, r\} \in] 1,2]$ the error bound

$$
\begin{gathered}
\left\|\mathbb{E}[u(\cdot, t)]-E_{M}\left[u_{\Delta}(\omega ; \cdot, t)\right]\right\|_{L^{q}\left(\Omega ; L^{p}(D)\right)} \leq C\left\{M^{1 / q-1}\left\|u_{0}\right\|_{L^{q}\left(\Omega ; L^{\max \{p, q\}}(D)\right)}\right. \\
+\bar{M}^{1-1 / p}\left\|u_{0}-u_{\Delta}(\cdot ; \cdot, 0)\right\|_{L^{q}\left(\Omega ; L^{p}(D)\right)}^{1 / p}+\Delta x^{1 /(3 p)} \bar{M}^{1-1 / p}(1+\bar{t})^{1 / p}\left\{\left\|A\left(u_{0}\right)_{x}\right\|_{L^{q}\left(\Omega ; L^{1}(D)\right)}^{1 / p}\right. \\
\left.\left.+\left\|\left(1+\|f(\omega ; \cdot)\|_{\text {Lip }}\right)\left|u_{0}\right|_{B V(D)}(\omega)\right\|_{L^{q}(\Omega)}^{1 / p}+\left\|\left|f\left(u_{0}\right)-A\left(u_{0}\right)_{x}\right|_{B V(D)}(\omega)\right\|_{L^{q}(\Omega)}^{1 / p}\right\}\right\} .
\end{gathered}
$$

where $C>0$ is independent of $M$ and of $\Delta x$ as $M \rightarrow \infty$ and as $\Delta x, \Delta t \downarrow 0$ and $\bar{M}:=\max \left\{M_{-}, M_{+}\right\}$as in (3.14).

Proof. The triangle inequality implies, for any $1 \leq p, q \leq \infty$

$$
\begin{aligned}
& \left\|\mathbb{E}[u(\cdot, t)]-E_{M}\left[u_{\Delta}(\omega ; \cdot, t)\right]\right\|_{L^{q}\left(\Omega ; L^{p}(D)\right)} \\
& \leq\left\|\mathbb{E}[u(\omega ; \cdot, t)]-E_{M}[u(\omega ; \cdot, t)]\right\|_{L^{q}\left(\Omega ; L^{p}(D)\right)} \\
& \quad+\left\|E_{M}[u(\omega ; \cdot, t)]-E_{M}\left[u_{\Delta}(\omega ; \cdot, t)\right]\right\|_{L^{q}\left(\Omega ; L^{p}(D)\right)} .
\end{aligned}
$$

For $p<q$, we estimate the first term in the upper bound of (4.18) with Hölder's inequality

$$
\left\|\mathbb{E}[u(\omega ; \cdot, t)]-E_{M}[u(\omega ; \cdot, t)]\right\|_{L^{q}\left(\Omega ; L^{p}(D)\right)} \leq C_{D}\left\|\mathbb{E}[u(\omega ; \cdot, t)]-E_{M}[u(\omega ; \cdot, t)]\right\|_{L^{q}\left(\Omega ; L^{q}(D)\right)} .
$$

By Theorem 4.1, we have

$$
\left\|\mathbb{E}[u(\omega ; \cdot, t)]-E_{M}[u(\omega ; \cdot, t)]\right\|_{L^{q}\left(\Omega ; L^{\tilde{p}}(D)\right)} \leq C M^{1 / q-1}\left\|u_{0}\right\|_{L^{q}\left(\Omega ; L^{\tilde{p}}(D)\right)},
$$

where $\widetilde{p}=\max \{q, p\}$. Hence, we arrive at

$$
\left\|\mathbb{E}[u(\omega ; \cdot, t)]-E_{M}[u(\omega ; \cdot, t)]\right\|_{L^{q}\left(\Omega ; L^{p}(D)\right)} \leq C_{D} M^{1 / q-1}\left\|u_{0}\right\|_{L^{q}\left(\Omega ; L^{p t}(D)\right)} .
$$


For the second term in the upper bound (4.18), we use the linearity of the estimator $E_{M}[]$ and the triangle inequality, and Remarks $4.3,4.5$ to obtain

$$
\begin{aligned}
& \left\|E_{M}[u(\omega ; \cdot, t)]-E_{M}\left[u_{\Delta}(\omega ; \cdot, t)\right]\right\|_{L^{q}\left(\Omega ; L^{p}(D)\right)} \\
& \quad \leq\left\|u(\cdot, t)-u_{\Delta}(\cdot, t)\right\|_{L^{q}\left(\Omega ; L^{p}(D)\right)} \\
& \leq\left\|u_{\Delta}\left(\cdot, t_{n}\right)-u\left(\cdot, t_{n}\right)\right\|_{L^{q}\left(\Omega ; L^{1}(D)\right)}^{1 / p}\left\|u_{\Delta}\left(\cdot, t_{n}\right)-u\left(\cdot, t_{n}\right)\right\|_{L^{q}\left(\Omega ; L^{\infty}(D)\right)}^{1-1 / p} \\
& \leq C \bar{M}^{1-1 / p}\left(\left\|u_{0}-u_{\Delta}(\cdot ; \cdot, 0)\right\|_{L^{q}\left(\Omega ; L^{p}(D)\right)}^{1 / p}+\Delta x^{1 /(3 p)}(1+\bar{t})^{1 / p}\left\{\left\|A\left(u_{0}\right)_{x}\right\|_{L^{q}\left(\Omega ; L^{1}(D)\right)}^{1 / p}\right.\right. \\
& \left.\left.\quad \quad+\left\|\left(1+\|f(\omega ; \cdot)\|_{L i p}\right)\left|u_{0}\right|_{B V(D)}(\omega)\right\|_{L^{q}(\Omega)}^{1 / p}+\left\|\left|f\left(u_{0}\right)-A\left(u_{0}\right)_{x}\right|_{B V(D)}(\omega)\right\|_{L^{q}(\Omega)}^{1 / p}\right\}\right),
\end{aligned}
$$

where the last inequality follows from the error estimate for the finite difference scheme, Proposition 4.4. Combining the two, we obtain the estimate (4.17).

\subsubsection{Work estimates}

We have seen that the computational work to obtain $\left\{u_{\Delta}(\cdot, t)\right\}_{0 \leq t \leq T}$, computed by the explicit or implicit scheme respectively, is asymptotically, as $\Delta x, \Delta t \rightarrow 0$, bounded as

$$
W_{\Delta}^{\mathrm{ex}} \leq C \Delta x^{-11 / 3}, \quad W_{\Delta}^{\mathrm{im}} \leq C \Delta x^{-2} \log \left(\Delta x^{-1}\right),
$$

which implies that the work for the computation of the MC estimate $E_{M}\left[u_{\Delta}(\cdot, t)\right]$ is of order

$$
W_{\Delta, M}^{\mathrm{ex}} \leq C M \Delta x^{-11 / 3}, \quad W_{\Delta, M}^{\mathrm{im}} \leq C M \Delta x^{-2} \log \left(\Delta x^{-1}\right),
$$

so that we obtain from (4.17) the convergence order in terms of work: To this end we equilibrate in (4.17) the two bounds by choosing $M^{(1-q) / q} \sim \Delta x^{1 /(3 p)}$, i.e. $M=C \Delta x^{\frac{q}{3 p(1-q)}}$. Inserting in (4.19) yields

$$
W_{\Delta, M}^{\mathrm{ex}} \leq C \Delta x^{-\frac{q+11 p(q-1)}{3 p(q-1)}}, \quad W_{\Delta, M}^{\mathrm{im}} \leq C \Delta x^{-\frac{q+6 p(q-1)}{3 p(q-1)}} \log \left(\Delta x^{-1}\right),
$$

so that we obtain from (4.17)

$$
\begin{aligned}
\left\|\mathbb{E}[u(\cdot, t)]-E_{M}\left[u_{\Delta}(\cdot, t)\right]\right\|_{L^{q}\left(\Omega ; L^{p}(D)\right)} & \leq\left(C_{u_{0}, t, A, f}^{q}\right)^{1 / p} \Delta x^{\frac{1}{3 p}} \\
& \leq\left(C_{u_{0}, t, A, f}^{q}\right)^{1 / p}\left(W_{\Delta, M}^{\mathrm{ex}}\right)^{-\frac{q-1}{q+11 p(q-1)}} \\
\left\|\mathbb{E}[u(\cdot, t)]-E_{M}\left[u_{\Delta}(\cdot, t)\right]\right\|_{L^{q}\left(\Omega ; L^{p}(D)\right)} & \leq\left(C_{u_{0}, t, A, f}^{q}\right)^{1 / p}\left(W_{\Delta, M}^{\mathrm{im}}\left(\log \left(W_{\Delta, M}^{\mathrm{im}}\right)\right)^{-1}\right)^{-\frac{q-1}{q+6 p(q-1)}}
\end{aligned}
$$

where $C_{u_{0}, t, A, f}^{q}$ is given by

$$
\begin{aligned}
C_{u_{0}, t, A, f}^{q} & =C(1+t)\left\{\left\|\left(1+\|f(\omega ; \cdot)\|_{\text {Lip }}\right)\left|u_{0}\right|_{B V(D)}(\omega)\right\|_{L^{q}(\Omega)}\right. \\
& \left.+\left\|A\left(u_{0}\right)_{x}\right\|_{L^{q}}\left(\Omega ; L^{1}(D)\right)+\left\|\left|f\left(u_{0}\right)-A\left(u_{0}\right)_{x}\right|_{B V(D)}(\omega)\right\|_{L^{q}}(\Omega)\right\} .
\end{aligned}
$$


Estimates (4.20) hold for $q=\min \{2, r\}$ as we used Remark 2.3 in its derivation. Assuming $r \geq 2$ in (3.20), we may optimize the bound (4.20a) with respect to $q$ for any $p \in[1, \infty[$. For $1<q \leq 2$, the function $q \mapsto q /(q-1)$ is monotonically decreasing. Therefore,

$$
\max _{1<q \leq 2} \frac{q-1}{q+11 p(q-1)}=\max _{1<q \leq 2} \frac{1}{q /(q-1)+11 p}
$$

is attained for $\tilde{q}=\min \{2, r\}$ resulting at best (for $r \geq 2$ ) in the error vs. work rate $1 /(2+11 p)$ in $(4.20 \mathrm{a})$, and $1 /(2+6 p)$ for the implicit scheme in (4.20b). On the other hand, in the deterministic case the convergence rates with respect to work read

$$
\begin{aligned}
& \left\|u(\cdot, t)-u_{\Delta}(\cdot, t)\right\|_{L^{p}(D)} \leq C_{T}^{1 / p} \Delta x^{1 /(3 p)} \leq C_{T}^{1 / p}\left(W_{\Delta}^{\mathrm{ex}}\right)^{-1 /(11 p)}, \\
& \left\|u(\cdot, t)-u_{\Delta}(\cdot, t)\right\|_{L^{p}(D)} \leq C_{T}^{1 / p}\left(W_{\Delta}^{\mathrm{im}}\left(\log \left(W_{\Delta}^{\mathrm{im}}\right)\right)^{-1}\right)^{-1 /(6 p)} .
\end{aligned}
$$

\subsection{Multilevel MC-FDM}

We next present and analyze a scheme that allows us to achieve almost the accuracy versus work bound (4.22) of the deterministic FDM also for the stochastic data $\left(u_{0}, f, A\right)$ satisfying (3.14) - (3.20), rather than the single level MC-FDM error bound (4.20). The key ingredient in the Multilevel Monte Carlo Finite Difference (MLMC-FDM) scheme is simultaneous MC sampling on different levels of resolution of the FDM, with level dependent numbers $M_{\ell}$ of $M C$ samples. To define these, we introduce some notation.

\subsubsection{Notation}

The MLMC-FDM is defined as a multilevel discretization in $x$ and $t$ with level dependent numbers $M_{\ell}$ of samples. To this end, we assume we are given a family of nested grids with cell sizes

$$
\Delta x_{\ell}=2^{-\ell} \Delta x_{0}, \quad \ell \in \mathbb{N}_{0},
$$

for some $\Delta x_{0}>0$. Similarly, we denote,

$$
\Delta t_{\ell}=C \Delta x_{\ell}^{8 / 3}
$$

the size of the time step for the explicit scheme corresponding to grid size $\Delta x_{\ell}$ and

$$
\Delta t_{\ell}=\theta \Delta x_{\ell}
$$

the size of the time step for the implicit scheme at level $\ell$. We denote by $u_{\ell}$ the approximation to (4.3) computed by (4.6) or (4.5) on the grid with cell and time step size $\Delta_{\ell}:=\left(\Delta x_{\ell}, \Delta t_{\ell}\right)$. 


\subsubsection{Derivation of $M L M C-F D M$}

As in plain MC-FDM, our aim is to estimate, for $0<t<\infty$, the expectation (or "ensemble average") $\mathbb{E}[u(\cdot, t)]$ of the random entropy solution of $(3.22)$ with random data $\left(u_{0}, f, A\right)(\omega), \omega \in \Omega$, satisfying (3.13) - (3.20) for sufficiently large values of $k$ (to be specified in the sequel). As in the previous section, $\mathbb{E}[u(\cdot, t)]$ will be estimated by replacing $u(\cdot, t)$ by a FDM approximation.

We generate a sequence of approximations, $\left\{u_{\ell}(\cdot, t)\right\}_{\ell=0}^{\infty}$ on the nested meshes with cell sizes $\Delta x_{\ell}$, time steps of sizes $\Delta t_{\ell}$. In the following we set $u_{-1}(\cdot, t):=0$. Then, given a target level $L \in \mathbb{N}$ of spatial resolution, we have

$$
\mathbb{E}\left[u_{L}(\cdot, t)\right]=\mathbb{E}\left[\sum_{\ell=0}^{L}\left(u_{\ell}(\cdot, t)-u_{\ell-1}(\cdot, t)\right)\right]
$$

We next estimate each term in (4.24) statistically by a MCM with a level-dependent number of samples, $M_{\ell}$; this gives the MLMC-FDM estimator

$$
E^{L}[u(\cdot, t)]=\sum_{\ell=0}^{L} E_{M_{\ell}}\left[u_{\ell}(\cdot, t)-u_{\ell-1}(\cdot, t)\right]
$$

where $E_{M}\left[u_{\Delta}(\cdot, t)\right]$ is as in (4.16), and where $u_{\ell}(\cdot, t)$ is computed on the mesh with grid size $\Delta x_{\ell}$ and time step $\Delta t_{\ell}$.

\subsubsection{Convergence Analysis}

We analyze the MLMC-FDM mean field error

$$
\left\|\mathbb{E}[u(\cdot, t)]-E^{L}[u(\cdot, t)]\right\|_{L^{q}\left(\Omega ; L^{p}(D)\right)}
$$

for $0<t<\infty, q \in] 1,2], p \in[1, \infty]$ and $L \in \mathbb{N}$. In particular, we are interested in the choice of the sample sizes $\left\{M_{\ell}\right\}_{\ell=0}^{\infty}$ such that, for every $L \in \mathbb{N}$, the MLMC error (4.26) is minimized. The principal issue in the design of MLMC-FDM is the optimal choice of $\left\{M_{\ell}\right\}_{\ell=0}^{\infty}$ such that, for each $L$, an error (4.26) is achieved with minimal total work given by (based on (4.19)),

$$
\begin{aligned}
W_{L, M L M C}^{\mathrm{ex}} & =C \sum_{\ell=0}^{L} M_{\ell} W_{\Delta_{\ell}}^{\mathrm{ex}}=\mathcal{O}\left(\sum_{\ell=0}^{L} M_{\ell} \Delta x_{\ell}^{-11 / 3}\right), \\
W_{L, M L M C}^{\mathrm{im}} & =C \sum_{\ell=0}^{L} M_{\ell} W_{\Delta_{\ell}}^{\mathrm{im}}=\mathcal{O}\left(\sum_{\ell=0}^{L} M_{\ell} \Delta x_{\ell}^{-2}\left|\log \left(\Delta x_{\ell}\right)\right|\right) .
\end{aligned}
$$

To estimate (4.26), we write (recall that $u_{-1}:=0$ ) using the triangle inequality, the linearity of the mathematical expectation $\mathbb{E}[\cdot]$ and the definition (4.25) of the MLMC estimator

$$
\begin{aligned}
\| \mathbb{E}[u(\cdot, t)]- & E^{L}[u(\cdot, t)] \|_{L^{q}\left(\Omega ; L^{p}(D)\right)}^{q} \\
& \leq C_{q}\left\|\mathbb{E}[u(\cdot, t)]-\mathbb{E}\left[u_{L}(\cdot, t)\right]\right\|_{L^{q}\left(\Omega ; L^{p}(D)\right)}^{q}
\end{aligned}
$$




$$
\begin{aligned}
& \quad+C_{q}\left\|\mathbb{E}\left[u_{L}(\cdot, t)\right]-E^{L}[u(\cdot, t)]\right\|_{L^{q}\left(\Omega ; L^{p}(D)\right)}^{q} \\
& =C_{q}\left\|\mathbb{E}[u(\cdot, t)]-\mathbb{E}\left[u_{L}(\cdot, t)\right]\right\|_{L^{q}\left(\Omega ; L^{p}(D)\right)}^{q} \\
& \quad+C_{q}\left\|\sum_{\ell=0}^{L} \mathbb{E}\left[u_{\ell}-u_{\ell-1}\right]-E_{M_{\ell}}\left[u_{\ell}-u_{\ell-1}\right]\right\|_{L^{q}\left(\Omega ; L^{p}(D)\right)}^{q} \\
& =: \mathrm{I}+\mathrm{II}
\end{aligned}
$$

We estimate terms I and II separately. By linearity of the expectation, term I equals

$$
\mathrm{I}=C_{q}\left\|\mathbb{E}\left[u(\cdot, t)-u_{L}(\cdot, t)\right]\right\|_{L^{q}\left(\Omega ; L^{p}(D)\right)}^{q} \leq C_{q}\left\|u(\cdot, t)-u_{L}(\cdot, t)\right\|_{L^{1}\left(\Omega ; L^{p}(D)\right)}^{q}
$$

As the function $\mathbb{E}\left[u(\cdot, t)-u_{L}(\cdot, t)\right]$ is deterministic, the $L^{q}\left(\Omega ; L^{p}(D)\right)$-norm is bounded by the $L^{1}\left(\Omega ; L^{p}(D)\right)$-norm due to $P(\Omega)=1$. Term I is therefore of the order of the deterministic FV discretization error that can be bounded by (4.15) with $r=1$ (cf. Remarks 4.3, 4.5 for $p>1$ ). We hence focus on term II. For $p<q$, we have that

$$
\begin{aligned}
& \| \sum_{\ell=0}^{L} \mathbb{E}\left[u_{\ell}-u_{\ell-1}\right]-E_{M_{\ell}}\left[u_{\ell}-u_{\ell-1}\right] \|_{L^{q}\left(\Omega ; L^{p}(D)\right)}^{q} \\
& \leq|D|^{(q-p) / p}\left\|\sum_{\ell=0}^{L} \mathbb{E}\left[u_{\ell}-u_{\ell-1}\right]-E_{M_{\ell}}\left[u_{\ell}-u_{\ell-1}\right]\right\|_{L^{q}\left(\Omega ; L^{q}(D)\right)}^{q},
\end{aligned}
$$

hence

$$
\mathrm{II} \leq C_{D}\left\|\sum_{\ell=0}^{L} \mathbb{E}\left[u_{\ell}-u_{\ell-1}\right]-E_{M_{\ell}}\left[u_{\ell}-u_{\ell-1}\right]\right\|_{L^{q}\left(\Omega ; L^{\tilde{p}}(D)\right)}^{q},
$$

where $\widetilde{p}=\max \{p, q\}$. The bound for term II can be written as

$$
\begin{aligned}
\mathrm{II} \leq C_{D} \| \sum_{\ell=0}^{L} \sum_{i=1}^{M_{\ell}} \frac{1}{M_{\ell}}\left(\mathbb{E}\left[u_{\ell}-u_{\ell-1}\right]-\left(\widehat{u}_{\ell}^{i}-\widehat{u}_{\ell-1}^{i}\right)\right) & \|_{L^{q}\left(\Omega ; L^{\tilde{p}}(D)\right)}^{q} \\
& =: C_{D}\left\|\sum_{\ell=0}^{L} \sum_{i=1}^{M_{\ell}} Y_{i, \ell}\right\|_{L^{q}\left(\Omega ; L^{\tilde{p}}(D)\right)}^{q}
\end{aligned}
$$

where $Y_{i, \ell}:=\frac{1}{M_{\ell}}\left(\mathbb{E}\left[u_{\ell}-u_{\ell-1}\right]-\left(\widehat{u}_{\ell}^{i}-\widehat{u}_{\ell-1}^{i}\right)\right), i=1, \ldots, M_{\ell}, \ell=0, \ldots, L$. The $Y_{i, \ell}$ are independent, mean zero random variables. Since $\widetilde{p}=\max \{p, q\}, L^{\widetilde{p}}(D)$ is of type $q \in] 1,2]$ (cf. Remark 2.3 (iii)). Therefore we can apply Proposition 2.4 to 
the finite sum $\sum_{\ell=0}^{L} \sum_{i=1}^{M_{\ell}} Y_{i, \ell}$ (first inequality in the following calculation):

$$
\begin{aligned}
\left\|\sum_{\ell=0}^{L} \sum_{i=1}^{M_{\ell}} Y_{i, \ell}\right\|_{L^{q}\left(\Omega ; L^{\tilde{p}}(D)\right)}^{q} & \leq\left(2 C_{t}\right)^{q} \sum_{\ell=0}^{L} \sum_{i=1}^{M_{\ell}}\left\|Y_{i, \ell}\right\|_{L^{q}\left(\Omega ; L^{\tilde{p}}(D)\right)}^{q} \\
& =\left(2 C_{t}\right)^{q} \sum_{\ell=0}^{L} M_{\ell}\left\|Y_{1, \ell}\right\|_{L^{q}\left(\Omega ; L^{\tilde{p}}(D)\right)}^{q} \\
& =\left(2 C_{t}\right)^{q} \sum_{\ell=0}^{L} M_{\ell}\left\|\frac{1}{M_{\ell}}\left(\mathbb{E}\left[u_{\ell}-u_{\ell-1}\right]-\left(\widehat{u}_{\ell}^{1}-\widehat{u}_{\ell-1}^{1}\right)\right)\right\|_{L^{q}\left(\Omega ; L^{\tilde{p}}(D)\right)}^{q} \\
& =\left(2 C_{t}\right)^{q} \sum_{\ell=0}^{L} M_{\ell}^{1-q}\left\|\mathbb{E}\left[u_{\ell}-u_{\ell-1}\right]-\left(u_{\ell}-u_{\ell-1}\right)\right\|_{L^{q}\left(\Omega ; L^{\tilde{p}}(D)\right)}^{q} \\
& \leq\left(2 C_{t}\right)^{q} \sum_{\ell=0}^{L} M_{\ell}^{1-q}\left\|u_{\ell}-u_{\ell-1}\right\|_{L^{q}\left(\Omega ; L^{\tilde{p}}(D)\right)}^{q} .
\end{aligned}
$$

We estimate for every $\ell \geq 0$ the size of the detail $u_{\ell}-u_{\ell-1}$ with the triangle inequality

$\left\|u_{\ell}(\cdot, t)-u_{\ell-1}(\cdot, t)\right\|_{L^{q}\left(\Omega ; L^{\tilde{p}}(D)\right)} \leq\left\|u(\cdot, t)-u_{\ell}(\cdot, t)\right\|_{L^{q}\left(\Omega ; L^{\tilde{p}}(D)\right)}+\left\|u(\cdot, t)-u_{\ell-1}(\cdot, t)\right\|_{L^{q}\left(\Omega ; L^{\tilde{p}}(D)\right)}$.

Combining this with (4.14), (4.15) with $\bar{t}=t, r=q$, (4.21) and (4.23), we obtain for every $\ell \in \mathbb{N}$ the estimate (using Hölder's inequality to get from $L^{\widetilde{p}}(D)$ to $L^{1}(D)$, cf. Remark 4.3, 4.5)

$$
\begin{aligned}
& \left\|\left(u_{\ell}-u_{\ell-1}\right)(\cdot, t)\right\|_{L^{q}\left(\Omega ; L^{\tilde{p}}(D)\right)} \\
& \quad \leq C \bar{M}^{1-1 / \widetilde{p}}\left(\left\|u_{0}-u_{\ell}(\cdot ; \cdot, 0)\right\|_{L^{q}\left(\Omega ; L^{1}(D)\right)}^{1 / \widetilde{p}}+\left\|u_{0}-u_{\ell-1}(\cdot ; \cdot, 0)\right\|_{L^{q}\left(\Omega ; L^{1}(D)\right)}^{1 / \widetilde{p}}\right) \\
& \quad+\left(C_{u_{0}, t, A, f}^{q}\right)^{1 / \widetilde{p}} \bar{M}^{1-1 / \widetilde{p}} \Delta x_{\ell}^{1 /(3 \widetilde{p})}
\end{aligned}
$$

where $\bar{M}=\max \left\{\left|M_{-}\right|,\left|M_{+}\right|\right\}$, cf. (3.14).

We use that the initial approximations $u_{\ell}(\cdot ; \cdot, 0)$ of $u(\cdot ; \cdot, 0)$ satisfy for any $1 \leq$ $r \leq \infty$ to get

$$
\left\|u(\cdot ; \cdot, 0)-u_{\ell}(\cdot ; \cdot, 0)\right\|_{L^{r}\left(\Omega ; L^{1}(D)\right)} \leq \Delta x_{\ell}\left\|\left|u_{0}\right|_{B V(D)}\right\|_{L^{r}(\Omega)} .
$$

Thus we can estimate the contribution of the errors of the approximation of the initial data by

$$
\begin{aligned}
\left\|u(\cdot ; \cdot, 0)-u_{\ell}(\cdot ; \cdot, 0)\right\|_{L^{q}\left(\Omega ; L^{1}(D)\right)}^{1 / \widetilde{p}} & \leq C\left(\Delta x_{\ell}\left\|\left|u_{0}\right|_{B V(D)}\right\|_{L^{q}(\Omega)}\right)^{1 / \widetilde{p}} \\
& \leq C \Delta x_{\ell}^{1 / \widetilde{p}}\left\|\left|u_{0}\right|_{B V(D)}\right\|_{L^{q}(\Omega)}^{1 / \widetilde{p}} .
\end{aligned}
$$

We insert this in (4.30) to obtain

$$
\left\|u_{\ell}(\cdot, t)-u_{\ell-1}(\cdot, t)\right\|_{L^{q}\left(\Omega ; L^{\tilde{p}}(D)\right)}
$$




$$
\begin{aligned}
\leq \bar{M}^{1-1 / \widetilde{p}} & \left(\left(C_{u_{0}, t, A, f}^{q}\right)^{1 / \widetilde{p}}+C \Delta x_{\ell}^{2 /(3 \widetilde{p})}\left\|\left|u_{0}\right|_{B V(D)}\right\|_{L^{q}(\Omega)}^{1 / \widetilde{p}}\right) \Delta x_{\ell}^{1 /(3 \widetilde{p})} \\
& \leq \bar{M}^{1-1 / \widetilde{p}}\left(C_{u_{0}, t, A, f}^{q}+\left\|\left|u_{0}\right|_{B V(D)}\right\|_{L^{q}(\Omega)}\right)^{1 / \widetilde{p}} \Delta x_{\ell}^{1 /(3 \widetilde{p})}
\end{aligned}
$$

where we used that $\Delta x_{\ell} \leq \mathcal{O}(1)$.

Theorem 4.8. Assume (3.14) - (3.20) for some $r>1$ and (4.23). Then, for any sequence $\left\{M_{\ell}\right\}_{\ell=0}^{\infty}$ of sample sizes at mesh level $\ell$, we have for the MLMC-FDM estimate $E^{L}[u(\cdot, t)]$ in (4.25) the error bound, for $q=\min \{r, 2\}$,

$$
\begin{aligned}
&\left\|\mathbb{E}[u(\cdot, t)]-E^{L}[u(\cdot, t)]\right\|_{L^{q}\left(\Omega ; L^{p}(D)\right)}^{q} \\
& \leq C \bar{M}^{q-q / p}\left\{\left(C_{u_{0}, t, A, f}^{1}\right)^{q} \Delta x_{L}^{q /(3 p)}+\Delta x_{L}^{q / p}\left\|\left|u_{0}\right|_{B V(D)}\right\|_{L^{1}(\Omega)}^{q}\right\} \\
&+C M_{0}^{1-q}\left\|u_{0}\right\|_{L^{q}\left(\Omega ; L^{\widetilde{p}}(D)\right)}^{q} \\
&+C \bar{M}^{q-q / \widetilde{p}}\left\{\sum_{\ell=0}^{L} M_{\ell}^{1-q} \Delta x_{\ell}^{q /(3 \widetilde{p})}\right\}\left(C_{u_{0}, t, A, f}^{q}+\left\|\left|u_{0}\right|_{B V(D)}\right\|_{L^{q}(\Omega)}\right)^{q / \widetilde{p}}
\end{aligned}
$$

where we have denoted $\widetilde{p}=\max \{p, q\}, \bar{M}=\max \left\{\left|M_{-}\right|,\left|M_{+}\right|\right\},(c f$. (3.14)) and

$$
\begin{aligned}
C_{u_{0}, t, A, f}^{j}=C(1+t)\{ & \left\|\left(1+\|f(\cdot ; \cdot)\|_{\text {Lip }}\right)\left|u_{0}\right|_{B V(D)}\right\|_{L^{j}(\Omega)} \\
& \left.+\left\|A\left(u_{0}\right)_{x}\right\|_{L^{j}\left(\Omega ; L^{1}(D)\right)}+\left\|\left|f\left(u_{0}\right)-A\left(u_{0}\right)_{x}\right|_{B V(D)}\right\|_{L^{j}(\Omega)}\right\},
\end{aligned}
$$

for $j=1, q$. In (4.31), $C>0$ is a constant that depends on the size of the domain $D$, but is independent of $L$, of $\left\{M_{\ell}\right\}_{\ell=0}^{L}$, and of the parameters $u_{0}, f, t$ and $A$.

Proof. We raise (4.30) to the $q$-th power, and insert the resulting estimate into the bound (4.29). The assertion (4.31) follows upon summing from $\ell=0, \ldots, L$, and using $\Delta x_{\ell} \leq \Delta x_{0} \leq \mathcal{O}(1)$ and adding the contribution from term I in (4.28).

\subsubsection{Determining the number of samples needed on each level}

The upper bound obtained in Theorem 4.8 is the basis for an optimization of the numbers $M_{\ell}$ of MC samples across the mesh levels. Our selection of the Monte Carlo sample sizes $M_{\ell}$ will be based on the last term in the error bound (4.31); we will use a Lagrange multiplier argument to determine the number of samples needed at each level in order to minimize the computational work given an error tolerance $\varepsilon$.

Lemma 4.9. Assume that $\Delta x_{\ell}=2^{-\ell} \Delta x_{0}$ for some $\Delta x_{0}>0$ and that the work scales aymptotically as in (4.27), i.e.,

$$
W_{L, M L M C}^{e x}=C \sum_{\ell=0}^{L} M_{\ell} \Delta x_{\ell}^{-w}, \quad W_{L, M L M C}^{i m}=C \sum_{\ell=0}^{L} M_{\ell} \Delta x_{\ell}^{-w} \log \left(\Delta x_{\ell}^{-1}\right),
$$


for $w>0$, where $w=11 / 3$ for the explicit scheme and $w=2$ for the implicit finite difference scheme. Assume furthermore that $L$ and $\Delta x_{0}$ are large enough such that $\Delta x_{L}^{s-w}>\Delta x_{0}^{-w}$. Given an error tolerance $\varepsilon>0$ and that the error at level $L$ scales as (cf. (4.31))

$$
\operatorname{Err}_{L}=C\left(\sum_{\ell=0}^{L} M_{\ell}^{1-q} \Delta x_{\ell}^{q s / \widetilde{p}}+\Delta x_{L}^{q s / p}+M_{0}^{1-q}\right) .
$$

Then, the optimal sample numbers $M_{\ell}$, with respect to the work measure (4.32) and with respect to the error bound (4.33), are given by

$$
\begin{aligned}
& M_{\ell}^{e x} \simeq M_{0}^{e x} \Delta x_{0}^{\frac{s}{\tilde{p}}} 2^{-\ell\left(\frac{s}{\bar{p}}+\frac{w}{q}\right)}, \\
& M_{\ell}^{i m} \simeq M_{0}^{i m} \frac{\Delta x_{0}^{\frac{s}{p}} 2^{-\ell\left(\frac{s}{\tilde{p}}+\frac{w}{q}\right)}}{\left(\ell+\log \left(\Delta x_{0}^{-1}\right)\right)^{\frac{1}{q}}}, \quad \ell=1, \ldots, L,
\end{aligned}
$$

where

$$
\begin{aligned}
& M_{0}^{e x} \simeq\left[\frac{1}{\varepsilon-\Delta x_{0}^{\frac{q s}{p}} 2^{-\frac{q s L}{p}}}\left(1+\Delta x_{0}^{\frac{s}{p}} \sum_{j=1}^{L} 2^{j\left(\frac{(q-1) w}{q}-\frac{s}{\tilde{p}}\right)}\right)\right]^{\frac{1}{q-1}} \\
& M_{0}^{i m} \simeq\left[\frac{1}{\varepsilon-\Delta x_{0}^{\frac{q s}{p}} 2^{-\frac{q s L}{p}}}\left(\log \left(\Delta x_{0}^{-1}\right)^{\frac{q-1}{q}}+\Delta x_{0}^{\frac{s}{\tilde{p}}} \sum_{j=1}^{L}\left(j+\log \left(\Delta x_{0}^{-1}\right)\right)^{\frac{q-1}{q}} 2^{j\left(\frac{(q-1) w}{q}-\frac{s}{p}\right)}\right)\right]^{\frac{1}{q-1}}
\end{aligned}
$$

where the $\simeq$ indicates that this is the number of samples up to a constant which may depend on the data $\left(u_{0}, f, A\right)$ and the domain but not on $\ell$ and $L$. In particular, as $L \rightarrow \infty$, the error of the MLMC-FDM algorithm, (4.31) scales for $p=1, r=q=2$ with respect to work as

$$
\begin{aligned}
\left\|\mathbb{E}[u(\cdot, t)]-E^{L}[u(\cdot, t)]\right\|_{L^{2}\left(\Omega ; L^{1}(D)\right)}^{2} & \leq \widehat{C}_{u_{0}, t, A, f}\left(W_{L, M L M C}^{\mathrm{ex}}\right)^{-1 / 6} \\
\left\|\mathbb{E}[u(\cdot, t)]-E^{L}[u(\cdot, t)]\right\|_{L^{2}\left(\Omega ; L^{1}(D)\right)}^{2} & \leq \widehat{C}_{u_{0}, t, A, f}\left(W_{L, M L M C}^{\mathrm{im}}\left(\log \left(W_{L, M L M C}^{\mathrm{im}}\right)\right)^{-1}\right)^{-2 / 7} .
\end{aligned}
$$

Proof. From (4.27), under the assumption $\Delta x_{\ell}=2^{-\ell} \Delta x_{0}$ for some $\Delta x_{0}>0$, in space dimension $d=1$ the work scales as in (4.32), that is,

$$
\begin{aligned}
W_{L, M L M C}^{\mathrm{ex}} & =C \sum_{\ell=0}^{L} M_{\ell} \Delta x_{\ell}^{-w}=C \Delta x_{0}^{-w} \sum_{\ell=0}^{L} M_{\ell} 2^{w \ell} \\
W_{L, M L M C}^{\mathrm{im}} & =C \sum_{\ell=0}^{L} M_{\ell} \Delta x_{\ell}^{-w} \log \left(\Delta x_{\ell}^{-1}\right)=C \Delta x_{0}^{-w} \sum_{\ell=0}^{L} M_{\ell}\left(\ell+\log \left(\Delta x_{0}^{-1}\right)\right) 2^{w \ell},
\end{aligned}
$$

for $w=11 / 3$ in the case of the explicit scheme and $w=2$ for the implicit scheme. 
The bound for the multi-level error (4.31) with $L$ discretization levels reads, asymptotically as $L \rightarrow \infty$,

$$
\begin{aligned}
\operatorname{Err}_{L} & =C\left(\sum_{\ell=0}^{L} M_{\ell}^{1-q} \Delta x_{\ell}^{q s / \widetilde{p}}+\Delta x_{L}^{q s / p}+M_{0}^{1-q}\right) \\
& =C\left(M_{0}^{1-q}+\Delta x_{0}^{q s / \widetilde{p}} \sum_{\ell=0}^{L} M_{\ell}^{1-q} 2^{-q s \ell / \widetilde{p}}+\Delta x_{0}^{q s / p} 2^{-q s L / p}\right) \\
& =C\left(M_{0}^{1-q}+\Delta x_{0}^{q s / \widetilde{p}} \sum_{\ell=1}^{L} M_{\ell}^{1-q} 2^{-q s \ell / \widetilde{p}}+\Delta x_{0}^{q s / p} 2^{-q s L / p}\right) .
\end{aligned}
$$

We optimize error versus work assuming a generic convergence order $s>0$ of the FV scheme, bearing in mind that we will choose finally $s=1 / 3$, based on the convergence estimate (4.13).

Using a Lagrange multiplier $\lambda$, we get for $\mathcal{L}:=W-\lambda\left(\varepsilon-\operatorname{Err}_{L}\right)$ the first order conditions

$$
0=\frac{\partial \mathcal{L}}{\partial M_{\ell}}, \quad \ell=0, \ldots, L
$$

This means that (omitting the constants $C$ ) there exist constants $\lambda_{e x}$ and $\lambda_{i m}$ which are independent of $\ell$ (but may depend on $L$ ) such that, for $1<q \leq 2$,

$$
\begin{aligned}
\Delta x_{0}^{-w} 2^{\ell w} & =\lambda_{e x}(q-1) \Delta x_{0}^{q s / \widetilde{p}} M_{\ell}^{-q} 2^{-q \ell s / \widetilde{p}}, \quad \ell=1, \ldots, L, \\
\Delta x_{0}^{-w} & =\lambda_{e x}(q-1) M_{0}^{-q}, \quad \ell=0,
\end{aligned}
$$

for the explicit scheme and

$$
\begin{aligned}
\Delta x_{0}^{-w}\left(\ell+\log \left(\Delta x_{0}^{-1}\right)\right) 2^{\ell w} & =\lambda_{i m}(q-1) \Delta x_{0}^{q s / \widetilde{p}} M_{\ell}^{-q} 2^{-q \ell s / \widetilde{p}}, \quad \ell=1, \ldots, L, \\
\Delta x_{0}^{-w} \log \left(\Delta x_{0}^{-1}\right) & =\lambda_{i m}(q-1) M_{0}^{-q}, \quad \ell=0,
\end{aligned}
$$

for the implicit scheme. Since $q>1$, we may solve for the sample numbers:

$$
\begin{aligned}
& M_{\ell}^{\mathrm{ex}}=\left(\lambda_{\mathrm{ex}}(q-1)\right)^{\frac{1}{q}}\left(\Delta x_{0} 2^{-\ell}\right)^{\frac{s}{p}+\frac{w}{q}}, \quad M_{\ell}^{\mathrm{im}}=\left(\frac{\lambda_{\mathrm{im}}(q-1)}{\ell+\log \left(\Delta x_{0}^{-1}\right)}\right)^{\frac{1}{q}}\left(\Delta x_{0} 2^{-\ell}\right)^{\frac{s}{p}+\frac{w}{q}}, \\
& \text { for } \quad \ell=1, \ldots, L, \text { and } \\
& \qquad M_{0}^{\mathrm{ex}}=\left(\lambda_{\mathrm{ex}}(q-1)\right)^{\frac{1}{q}} \Delta x_{0}^{w / q}, \quad M_{0}^{\mathrm{im}}=\left(\frac{\lambda_{\mathrm{im}}(q-1)}{\log \left(\Delta x_{0}^{-1}\right)}\right)^{\frac{1}{q}} \Delta x_{0}^{w / q} .
\end{aligned}
$$

Using the constraint $\operatorname{Err}_{L}=\varepsilon$, we get for the prescribed accuracy $\varepsilon>0$,

$$
\begin{aligned}
& \varepsilon=\Delta x_{0}^{\frac{q s}{p}} 2^{-\frac{q s L}{p}}+\frac{\Delta x_{0}^{\frac{w(1-q)}{q}}}{\left(\lambda_{\operatorname{ex}}(q-1)\right)^{\frac{q-1}{q}}}\left(1+\Delta x_{0}^{\frac{s}{p}} \sum_{\ell=1}^{L} 2^{\ell\left(\frac{(q-1) w}{q}-\frac{s}{p}\right)}\right), \\
& \varepsilon=\Delta x_{0}^{\frac{q s}{p}} 2^{-\frac{q s L}{p}}+\frac{\Delta x_{0}^{\frac{w(1-q)}{q}}}{\left(\lambda_{\operatorname{im}}(q-1)\right)^{\frac{q-1}{q}}}\left(\log \left(\Delta x_{0}^{-1}\right)^{\frac{q-1}{q}}+\Delta x_{0}^{\frac{s}{p}} \sum_{\ell=1}^{L}\left(\ell+\log \left(\Delta x_{0}^{-1}\right)\right)^{\frac{q-1}{q}} 2^{\ell\left(\frac{(q-1) w}{q}-\frac{s}{p}\right)}\right) .
\end{aligned}
$$


We solve this for $\lambda_{\mathrm{ex}}, \lambda_{\mathrm{im}}$ respectively,

$$
\begin{aligned}
\lambda_{\mathrm{ex}}= & \frac{1}{\Delta x_{0}^{w}(q-1)}\left[\frac{1}{\varepsilon-\Delta x_{0}^{\frac{q s}{p}} 2^{-\frac{q s L}{p}}}\left(1+\Delta x_{0}^{\frac{s}{\tilde{p}}} \sum_{j=1}^{L} 2^{j\left(\frac{(q-1) w}{q}-\frac{s}{\tilde{p}}\right)}\right)\right]^{\frac{q}{q-1}}, \\
\lambda_{\mathrm{im}}= & \frac{1}{\Delta x_{0}^{w}(q-1)} \\
& \times\left[\frac{1}{\varepsilon-\Delta x_{0}^{\frac{q s}{p}} 2^{-\frac{q s L}{p}}}\left(\log \left(\Delta x_{0}^{-1}\right)^{\frac{q-1}{q}}+\Delta x_{0}^{\frac{s}{p}} \sum_{j=1}^{L}\left(j+\log \left(\Delta x_{0}^{-1}\right)\right)^{\frac{q-1}{q}} 2^{j\left(\frac{(q-1) w}{q}-\frac{s}{p}\right)}\right)\right]^{\frac{q}{q-1}},
\end{aligned}
$$

and insert it in the expressions for $M_{\ell}$, resulting in (4.34):

$$
\begin{aligned}
M_{\ell}^{\mathrm{ex}} \simeq & {\left[\frac{1}{\varepsilon-\Delta x_{0}^{\frac{q s}{p}} 2^{-\frac{q s L}{p}}}\left(1+\Delta x_{0}^{\frac{s}{p}} \sum_{j=1}^{L} 2^{j\left(\frac{(q-1) w}{q}-\frac{s}{p}\right)}\right)\right]^{\frac{1}{q-1}} \Delta x_{0}^{\frac{s}{p}} 2^{-\ell\left(\frac{s}{p}+\frac{w}{q}\right)}, } \\
M_{\ell}^{\mathrm{im}} \simeq & {\left[\frac{1}{\varepsilon-\Delta x_{0}^{\frac{q s}{p}} 2^{-\frac{q s L}{p}}}\left(\log \left(\Delta x_{0}^{-1}\right)^{\frac{q-1}{q}}+\Delta x_{0}^{\frac{s}{p}} \sum_{j=1}^{L}\left(j+\log \left(\Delta x_{0}^{-1}\right)\right)^{\frac{q-1}{q}} 2^{j\left(\frac{(q-1) w}{q}-\frac{s}{\bar{p}}\right)}\right)\right]^{\frac{1}{q-1}} } \\
& \times \frac{\Delta x_{0}^{\frac{s}{p}} 2^{-\ell\left(\frac{s}{\tilde{p}}+\frac{w}{q}\right)}}{\left(\ell+\log \left(\Delta x_{0}^{-1}\right)\right)^{\frac{1}{q}}}, \quad \ell=1, \ldots, L,
\end{aligned}
$$

where the $\simeq$ indicates that this is the number of samples up to a constant which may depend on the data $\left(u_{0}, f, A\right)$ and the domain but not on $\ell$ and $L$. For $M_{0}$, we obtain

$$
\begin{aligned}
& M_{0}^{\mathrm{ex}} \simeq\left[\frac{1}{\varepsilon-\Delta x_{0}^{\frac{q s}{p}} 2^{-\frac{q s L}{p}}}\left(1+\Delta x_{0}^{\frac{s}{p}} \sum_{j=1}^{L} 2^{j\left(\frac{(q-1) w}{q}-\frac{s}{p}\right)}\right)\right]^{\frac{1}{q-1}}, \\
& M_{0}^{\mathrm{im}} \simeq\left[\frac{1}{\varepsilon-\Delta x_{0}^{\frac{q s}{p}} 2^{-\frac{q s L}{p}}}\left(\log \left(\Delta x_{0}^{-1}\right)^{\frac{q-1}{q}}+\Delta x_{0}^{\frac{s}{p}} \sum_{j=1}^{L}\left(j+\log \left(\Delta x_{0}^{-1}\right)\right)^{\frac{q-1}{q}} 2^{j\left(\frac{(q-1) w}{q}-\frac{s}{p}\right)}\right)\right]^{\frac{1}{q-1}}
\end{aligned}
$$

Inserting into the work estimate (4.32), we obtain ther asymptotic work vs. accuracy relations

$$
\begin{aligned}
W_{L, M L M C}^{\mathrm{ex}} \simeq & {\left[\frac{1}{\varepsilon-\Delta x_{0}^{\frac{q s}{p}} 2^{-\frac{q s L}{p}}}\left(1+\Delta x_{0}^{\frac{s}{\tilde{p}}} \sum_{j=1}^{L} 2^{j\left(\frac{(q-1) w}{q}-\frac{s}{p}\right)}\right)\right]^{\frac{1}{q-1}} \Delta x_{0}^{-w}\left(1+\Delta x_{0}^{\frac{s}{p}} \sum_{\ell=1}^{L} 2^{\ell\left(\frac{w(q-1)}{q}-\frac{s}{\bar{p}}\right)}\right), } \\
W_{L, M L M C} \simeq & {\left[\frac{1}{\varepsilon-\Delta x_{0}^{\frac{q s}{p}} 2^{-\frac{q s L}{p}}}\left(\log \left(\Delta x_{0}^{-1}\right)^{\frac{q-1}{q}}+\Delta x_{0}^{\frac{s}{p}} \sum_{j=1}^{L}\left(j+\log \left(\Delta x_{0}^{-1}\right)\right)^{\frac{q-1}{q}} 2^{j\left(\frac{(q-1) w}{q}-\frac{s}{p}\right)}\right)\right]^{\frac{1}{q-1}} } \\
& \times \Delta x_{0}^{-w}\left(\log \left(\Delta x_{0}^{-1}\right)+\Delta x_{0}^{\frac{s}{p}} \sum_{\ell=1}^{L}\left(\ell+\log \left(\Delta x_{0}^{-1}\right)\right)^{\frac{q-1}{q}} 2^{\ell\left(\frac{w(q-1)}{q}-\frac{s}{\tilde{p}}\right)}\right) .
\end{aligned}
$$


Assuming that $r \geq 2$, we may choose $q=2$ and $p=1$ which implies $\tilde{p}=\max \{p, q\}=$ 2. With $s=1 / 3$ we obtain for the explicit scheme with $w=11 / 3$, that

$$
\frac{(q-1) w}{q}-\frac{s}{\widetilde{p}}=\frac{w-s}{2}=\frac{11}{6}-\frac{1}{6}=\frac{5}{3}>0
$$

and hence the terms $2^{\frac{L-s}{2}}$ dominate the sums in the expression for $W_{L, M L M C}^{\mathrm{ex}}$. Choosing $\varepsilon=2 \Delta x_{0}^{2 s} \cdot 2^{-2 s L} \simeq 2 \Delta x_{L}^{2 s}$, the work for the explicit MLMC scheme is thus of the order

$$
\begin{aligned}
W_{L, M L M C}^{\mathrm{ex}} & \simeq \Delta x_{0}^{-2 s} 2^{2 s L}\left(\Delta x_{0}^{-\frac{w}{2}}+\Delta x_{0}^{\frac{s-w}{2}} 2^{L \frac{w-s}{2}}\right)^{2} \\
& \simeq \Delta x_{0}^{-2 s} 2^{2 s L}\left(\Delta x_{0}^{-w}+\Delta x_{0}^{s-w} 2^{L(w-s)}\right) \\
& \simeq \Delta x_{L}^{-2 s}\left(\Delta x_{0}^{-w}+\Delta x_{L}^{s-w}\right)
\end{aligned}
$$

If $\Delta x_{L}^{s-w}>\Delta x_{0}^{-w}$, then this term is dominated by $\Delta x_{L}^{-(s+w)}$ and

$$
\varepsilon \simeq \Delta x_{L}^{2 s} \simeq\left(\Delta x_{L}^{-(s+w)}\right)^{-\frac{2 s}{s+w}} \simeq\left(W_{L, M L M C}^{\mathrm{ex}}\right)^{-\frac{2 s}{s+w}} .
$$

For the explicit scheme $\frac{2 s}{s+w}=1 / 6$.

For the implicit scheme, we have

$$
\begin{aligned}
W_{L, M L M C}^{\mathrm{im}} & \simeq \Delta x_{0}^{-2 s} 2^{2 s L}\left(\log \left(\Delta x_{0}^{-1}\right)^{3 / 4} \Delta x_{0}^{-\frac{w}{2}}+\Delta x_{0}^{\frac{s-w}{2}}\left(L+\log \left(\Delta x_{0}^{-1}\right)\right)^{1 / 2} 2^{L \frac{w-s}{2}}\right)^{2} \\
& \simeq \Delta x_{0}^{-2 s} 2^{2 s L}\left(\log \left(\Delta x_{0}^{-1}\right)^{3 / 2} \Delta x_{0}^{-w}+\Delta x_{0}^{s-w}\left(L+\log \left(\Delta x_{0}^{-1}\right)\right) 2^{L(w-s)}\right) \\
& \simeq \Delta x_{L}^{-2 s}\left(\log \left(\Delta x_{0}^{-1}\right)^{3 / 2} \Delta x_{0}^{-w}+\log \left(\Delta x_{L}^{-1}\right) \Delta x_{L}^{s-w}\right) .
\end{aligned}
$$

So if $\log \left(\Delta x_{0}^{-1}\right)^{3 / 2} \Delta x_{0}^{-w}<\log \left(\Delta x_{L}^{-1}\right) \Delta x_{L}^{s-w}$, the work is asymptotically dominated by

$$
W_{L, M L M C}^{\mathrm{im}} \simeq \Delta x_{L}^{-(s+w)} \log \left(\Delta x_{L}^{-1}\right),
$$

and we get,

$$
\varepsilon \simeq \Delta x_{L}^{2 s} \simeq\left(\Delta x_{L}^{-(s+w)}\right)^{-\frac{2 s}{s+w}} \simeq\left(W_{L, M L M C}^{\mathrm{im}} \log \left(W_{L, M L M C}^{\mathrm{im}}\right)^{-1}\right)^{-\frac{2 s}{s+w}} .
$$

Since $s=1 / 3$ and $w=2$ for the implicit scheme, the rate is $\frac{2 s}{s+w}=2 / 7$ up to the logarithmic factor.

Summing up, under Assumption 3.20 with $r \geq 2$ and for $p=1$, we have, as $L \rightarrow \infty$, the following error estimate of the MLMC method in terms of work

$$
\begin{aligned}
\left\|\mathbb{E}[u(\cdot, t)]-E^{L}[u(\cdot, t)]\right\|_{L^{2}\left(\Omega ; L^{1}(D)\right)}^{2} & \leq \widehat{C}_{u_{0}, t, A, f}\left(W_{L, M L M C}^{\mathrm{ex}}\right)^{-1 / 6} \\
\left\|\mathbb{E}[u(\cdot, t)]-E^{L}[u(\cdot, t)]\right\|_{L^{2}\left(\Omega ; L^{1}(D)\right)}^{2} & \leq \widehat{C}_{u_{0}, t, A, f}\left(W_{L, M L M C}^{\mathrm{im}}\left(\log \left(W_{L, M L M C}^{\mathrm{im}}\right)\right)^{-1}\right)^{-2 / 7} .
\end{aligned}
$$

Remark 4.10. This is worse than the error vs. work bounds (4.22) for the deterministic schemes for $p=1$ but an improved rate as compared to the single level 
Monte Carlo, c.f. (4.20). We conclude the analysis with the observation that in (4.36), we assumed the integrability condition (3.20) holds with some $r \geq 2$. If (3.20) holds with $1<r<2$, analogous error bounds will result from the foregoing analysis, albeit with more pessimistic error vs. work bounds.

\section{Numerical Experiments}

In this section, we will test the method on two examples motivated by two-phase flow in porous media ${ }^{a}$. In one space dimension, the time evolution of the water saturation $u \in[0,1]$ can be modeled by the conservation law

$$
u_{t}+f(u)_{x}=\left(a(u) u_{x}\right)_{x}, \quad(t, x) \in[0, T] \times \mathbb{R}, u(0, x)=u_{0}(x), \quad x \in \mathbb{R} .
$$

The functions $f$ and $a$ are of the form

$$
f(u)=\frac{\lambda^{\mathrm{w}}(u)}{\lambda^{\mathrm{w}}(u)+\lambda^{\mathrm{o}}(u)}, \quad a(u)=\frac{\lambda^{\mathrm{w}}(u) \lambda^{\mathrm{o}}(u)}{\lambda^{\mathrm{w}}(u)+\lambda^{\mathrm{o}}(u)} p_{c}^{\prime}(u),
$$

here $p_{c}^{\prime}$ denotes the derivative of the capillary pressure. In some models this derivative has the expression

$$
p_{c}^{\prime}(u)=-\left(u^{-\kappa / 3}-1\right)^{1 / \kappa}
$$

for a constant $\kappa \in(0,1)$, see [17]. The functions $\lambda^{\mathrm{w}}, \lambda^{\mathrm{o}}$ are the phase mobilities/relative permeabilities of the water and the oil phase respectively. The relative permeability of the water phase $\lambda^{\mathrm{w}}$ is a monotone function with $\lambda^{\mathrm{w}}(0)=0$, $\lambda^{\mathrm{w}}(1)=1$, and the relative permeability of the oil phase $\lambda^{\mathrm{o}}$ is a monotone decreasing function such that $\lambda^{\mathrm{o}}(0)=1$ and $\lambda^{\mathrm{o}}(1)=0$. Often one uses the simple expressions

$$
\lambda^{\mathrm{w}}(u)=u^{2}, \quad \lambda^{\mathrm{o}}(u)=(1-u)^{2} .
$$

Such a form of the relative permeability is of course a simplification, more accurate models are based on experiments. These functions therefore have some uncertainty associated with them. Hence it is natural to model the relative permeabilities as random variables.

Using (5.3) and (5.4) will yield an expression for $A(u)=\int a(u) d u$ that is costly to evaluate numerically since there is no closed form expression available. Therefore we use the expression

$$
A(u)=\frac{1}{282}\left(\frac{\lambda(u)}{\lambda^{\mathrm{w}}(u)+\lambda^{\mathrm{o}}(u)}\right)^{2} .
$$

This function is quite close to the diffusion function given by (5.2), (5.3) and (5.4) for $\kappa=4$. In our numerical experiments, we use the domains $D=(0,2)$ with periodic boundary conditions. Furthermore, we test only the implicit scheme. 
In the experiments we also indicate an estimator of the variance of the computed estimation of the mean. The estimate of the variance is calculated using the following formula

$$
\begin{aligned}
V_{L} & =\sum_{\ell=1}^{L} \Delta V_{\ell}+V_{0}, \\
\Delta V_{\ell} & =E_{M_{\ell}}\left[\left(u_{\ell}-u_{\ell-1}-E_{M_{\ell}}\left[u_{\ell}-u_{\ell-1}\right]\right)^{2}\right], \\
V_{0} & =E_{M_{0}}\left[\left(u_{0}-E_{M_{0}}\left[u_{0}\right]\right)^{2}\right] .
\end{aligned}
$$

When choosing the number of samples we use formulas (4.34) - (4.35) with "=" replacing " $\simeq$ ", and $p=1, r=q=2$ and $s=1 / 3$. If the resulting number is not an integer, we choose the number of samples to be the smallest integer greater than this number.

In order to compute an estimate of

$$
\mathbb{E}\left[\left\|E^{L}[u(\cdot, T)]-\mathbb{E}[u(\cdot, T)]\right\|_{L^{1}(D)}^{2}\right],
$$

we use the root mean square estimate

$$
\mathcal{R} \mathcal{M S}=\sqrt{\sum_{k=1}^{N}\left(\mathcal{R} \mathcal{M S}_{k}\right)^{2} / N},
$$

where

$$
\mathcal{R M S}_{k}=100 \times \frac{\left\|U_{\mathrm{ref}}(\cdot, T)-U_{k}(\cdot, T)\right\|_{L^{1}}}{\left\|U_{\mathrm{ref}}(\cdot, T)\right\|_{L^{1}}} .
$$

In [29], the sensitivity of the error with respect to the parameter $N$ is investigated. In the present numerical experiments, we use $N=10$ which was shown to be sufficient for most problems $[29,30]$. The reference approximation of $\mathbb{E}[u(T)], U_{\text {ref }}$, was computed by first computing an approximation $u_{\Delta}\left(\omega_{i} ; \cdot, T\right)$ to $u\left(\omega_{j} ; \cdot, T\right)$ for a large number of uniformly spaced points $\left\{\omega_{i}\right\}_{i=1}^{K}$ in $\Omega$ (which in our examples are a closed interval and a rectangle), and then finding $U_{\text {ref }}(\cdot, T)$ by applying the trapezoidal rule to approximate the integral $\int_{\Omega} u(\omega ; \cdot, T) d \omega$ using $\left\{u_{\Delta}\left(\omega_{i} ; \cdot, T\right)\right\}_{i=1}^{K}$.

The CFL constraint which ensures convergence of the nonlinear solver at each (implicit) timestep was (empirically) chosen as

$$
\max \{10 \Delta x, 0.0824 \log (\Delta x)+0.7286\} \text {. }
$$

This is the maximum of a linear interpolation in $\log (\Delta x)$ between $\left(\log \left(2^{-4}\right), 1 / 2\right)$ and $\left(\log \left(2^{-11}\right), 1 / 10\right)$, and $10 \Delta x$, and was found to be sufficient to ensure stability for these two numerial experiments.

\subsection{Random exponent}

For this example we will model the relative permeabilities by

$$
\lambda^{\mathrm{w}}(u)=u^{p(\omega)}, \quad \lambda^{\mathrm{o}}(u)=(1-u)^{p(\omega)},
$$


where the random exponent $p$ is uniformly distributed in the interval $[1.5,2.5]$. As initial data, we use

$$
u_{0}(x)= \begin{cases}0, & x \in[0,0.1) \cup[1,2), \\ 1, & x \in[0.1,1),\end{cases}
$$

and periodically extended outside $[0,2]$. Figure 1 shows a sample of the approximate random entropy solution with $p=2.13$ calculated using $2^{9}$ grid points, at time $T=0.5$, and an estimate of the mean $\mathbb{E}[s(\cdot, 0.5)]$ computed by the implicit multilevel Monte Carlo finite difference method with $\Delta x_{0}=2^{-4}$ and $L=5$, which gives $M_{0}=$ 4150. To compute the reference solution we approximated the expectation with
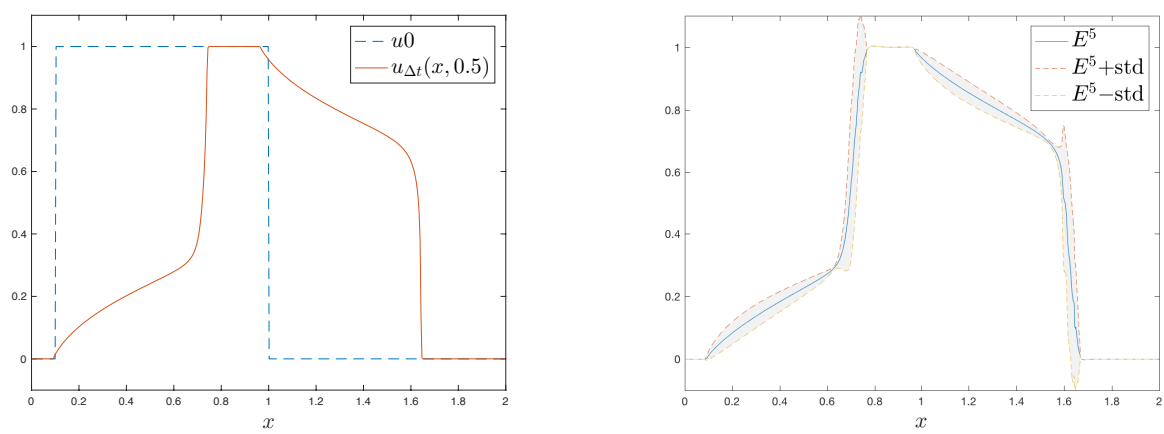

Fig. 1. Left: One sample of the random entropy solution of (5.1) with (5.8), (5.5) and (5.7) at time $T=0.5$ computed on a mesh with 512 points using the implicit scheme. Right: A sample of the estimator $E^{L}[u(\cdot, T)]$ for (5.1) with (5.8), (5.2) and (5.7) at time $T=0.5$ (solid line), the dashed lines denote $E^{L}[u(\cdot, T)] \pm$ standard deviation.

respect to the uniform probability measure in the interval $(1.5,2.5)$ by a trapezoidal rule with 200 equispaced grid points in $[1.5,2.5]$.

Table 1 shows the estimated $\mathcal{R} M S$ errors as a function of the number of levels $L$ for $L=1, \ldots, 6$ and $\Delta x_{0}=2^{-4}$. In addition we show the rates $r_{1}$ and $r_{2}$ based on a best linear fit under the assumptions that error $\sim\left(\Delta x_{L}\right)^{r_{1}}$ and error $\sim$ $(\text { time used })^{-r_{2}}$. We see that the rates, both with respect to error vs. mesh resolution

\begin{tabular}{c|cccccc|c}
$L$ & 1 & 2 & 3 & 4 & 5 & 6 & rate \\
\hline$\Delta x_{L}$ & $2^{-5}$ & $2^{-6}$ & $2^{-7}$ & $2^{-8}$ & $2^{-9}$ & $2^{-10}$ & \\
$\mathcal{R} \mathcal{M S}$ & 10.5 & 5.0 & 3.0 & 1.5 & 0.6 & 0.3 & 1.04 \\
run time (s) & 1 & 4 & 16 & 73 & 321 & 1405 & 0.49
\end{tabular}

Table $1 . \mathcal{R} \mathcal{M S}$ vs. $L$ for experiment 1.

at level $L$ and with respect to error vs. work (in this case crudely measured via the 
run time), are better than what theory predicts.

\subsection{Random residual saturation}

In the following numerical example, we will model the relative permeabilities by two random variables

$$
\lambda^{\mathrm{w}}(u)=\mathbf{1}_{u>u_{\mathrm{w}}^{*}\left(\omega_{1}\right)}(u) \frac{\left(u-u_{\mathrm{w}}^{*}\left(\omega_{1}\right)\right)^{2}}{\left(1-u_{\mathrm{w}}^{*}\left(\omega_{1}\right)\right)^{2}}, \quad \lambda^{\mathrm{o}}(u)=\mathbf{1}_{u \leq u_{\mathrm{o}}^{*}\left(\omega_{2}\right)}(u)\left(1-\frac{u}{u_{\mathrm{o}}^{*}\left(\omega_{2}\right)}\right)^{2},
$$

with $\quad u_{\mathrm{w}}^{*}\left(\omega_{1}\right) \sim \mathcal{U}(0.0,0.3), \quad u_{\mathrm{o}}^{*}\left(\omega_{2}\right) \sim \mathcal{U}(0.7,1.0), \quad u_{\mathrm{w}}^{*}\left(\omega_{1}\right) \perp u_{\mathrm{o}}^{*}\left(\omega_{2}\right)$,

that is, we assume that the residual saturations $u_{\mathrm{w}}^{*}, u_{\mathrm{o}}^{*}$ are independent, uniformly distributed random variables. As initial data, we use again (5.8) with periodic boundary conditions.

The resulting $(f, A)\left(\omega_{1}, \omega_{2} ; \cdot\right)$ again satisfies assumptions $(3.13)-(3.19)$, so that the random entropy solution from Definition 3.6 exists and Theorems 3.7, 4.8 apply. In Figure 2 on the left hand side, we have plotted a sample $u(\omega ; T, \cdot)$ of the random entropy solution at time $T=0.5$ and on the right hand side we have plotted a sample of the MLMC-FDM estimator $E^{L}(u(T))$ for $L=5, \Delta x_{0}=2^{-4}$. To
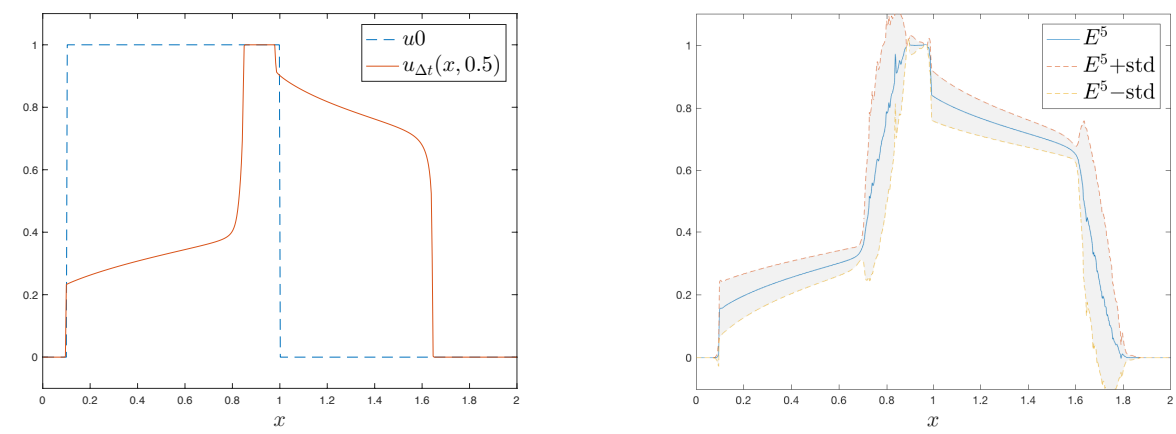

Fig. 2. Left: One sample of the random entropy solution of (5.1) with (5.8), (5.2) and (5.9) at time $T=0.5$ computed on a mesh with 512 points. Right: A sample of the estimator $E^{5}[u(\cdot, T)]$ for (5.1) with (5.8), (5.2) and (5.9) at time $T=0.5$ (solid line), the dashed lines show $E^{5}[u(\cdot, T)] \pm$ standard deviation.

compute the reference solution in this case we approximated the expectation with respect to the uniform probability measure over the rectangle $[0,0.3] \times[0.7,1.0]$ by a tensorized trapezoidal rule with $60 \times 60$ uniformly spaced points in the rectangle $[0,0.3] \times[0.7,1.0]$. Table 2 shows the estimated errors $\mathcal{R} \mathcal{M S}$ calculated using (5.6) as a function of $L$. Again, we observe that the numerical convergence rates are larger than the theoretical bounds. 


\begin{tabular}{c|cccccc|c}
$L$ & 1 & 2 & 3 & 4 & 5 & 6 & rate \\
\hline$\Delta x_{L}$ & $2^{-5}$ & $2^{-6}$ & $2^{-7}$ & $2^{-8}$ & $2^{-9}$ & $2^{-10}$ & \\
$\mathcal{R M S}$ & 6.9 & 3.6 & 2.2 & 1.2 & 0.7 & 0.5 & 0.75 \\
run time (s) & 2 & 11 & 60 & 227 & 628 & 2457 & 0.37
\end{tabular}

Table 2. $\mathcal{R M S}$ vs. $L$ for experiment 2 .

\section{Acknowledgments}

We thank Kjetil Olsen Lye for thorough proofreading.

\section{References}

[1] M. Afif and B. Amaziane. Convergence of finite volume schemes for a degenerate convection-diffusion equation arising in flow in porous media. Comput. Methods Appl. Mech. Engrg., 191(46):5265-5286, 2002.

[2] F. Bouchut, F. R. Guarguaglini, and R. Natalini. Diffusive bgk approximations for nonlinear multidimensional parabolic equations. Indiana Univ. Math. J., 49(2):723749, 2000.

[3] J. Boussinesq. Recherches théoriques sur l'écoulement des nappes d'eau infiltrées dans le sol et sur le débit des sources. Journal de mathématiques pures et appliquées, 10:5-78, 1904.

[4] J. Carrillo. Entropy solutions for nonlinear degenerate problems. Arch. Ration. Mech. Anal., 147(4):269-361, 1999.

[5] G.-Q. Chen and B. Perthame. Well-posedness for non-isotropic degenerate parabolichyperbolic equations. Ann. Inst. H. Poincaré Anal. Non Linéaire, 20(4):645-668, 2003.

[6] B. Cockburn and G. Gripenberg. Continuous dependence on the nonlinearities of solutions of degenerate parabolic equations. Journal of differential equations, 151(2):231$251,1999$.

[7] B. Cockburn and C.-W. Shu. The local discontinuous Galerkin method for timedependent convection-diffusion systems. SIAM J. Numer. Anal., 35(6):2440-2463, 1998.

[8] C. M. Dafermos. Hyperbolic conservation laws in continuum physics, volume 325 of Grundlehren der Mathematischen Wissenschaften [Fundamental Principles of Mathematical Sciences]. Springer-Verlag, Berlin, third edition, 2010.

[9] S. Evje and K. H. Karlsen. Degenerate convection-diffusion equations and implicit monotone difference schemes. In Hyperbolic problems: theory, numerics, applications, Vol. I (Zürich, 1998), volume 129 of Internat. Ser. Numer. Math., pages 285-294. Birkhäuser, Basel, 1999.

[10] S. Evje and K. H. Karlsen. Viscous splitting approximation of mixed hyperbolicparabolic convection-diffusion equations. Numerische Mathematik, 83(1):107-137, 1999.

[11] S. Evje and K. H. Karlsen. Monotone difference approximations of BV solutions to degenerate convection-diffusion equations. SIAM J. Numer. Anal., 37(6):1838-1860, 2000 .

[12] S. Evje and K. H. Karlsen. An error estimate for viscous approximate solutions of degenerate parabolic equations. J. Nonlinear Math. Phys., 9(3):262-281, 2002.

[13] S. Evje, K. H. Karlsen, and N. H. Risebro. A Continuous Dependence Result For 
Nonlinear Degenerate Parabolic Equations With Spatially Dependent Flux Function. In Proc. Hyp, pages 337-346, 2000.

[14] R. Eymard, T. Gallouët, and R. Herbin. Convergence of a finite volume scheme for nonlinear degenerate parabolic equations. Numerische Mathematik, 92:41-82, 2002.

[15] R. Eymard, T. Gallouët, and R. Herbin. Error estimate for approximate solutions of a nonlinear convection-diffusion problem. Advances in Differential Equations, 7(4):419440, 2002.

[16] E. Godlewski and P.-A. Raviart. Hyperbolic systems of conservation laws, volume 3/4 of Mathématiques 85 Applications (Paris) [Mathematics and Applications]. Ellipses, Paris, 1991.

[17] R. Helmig, A. Weiss, and B. Wohlmuth. Dynamic capillary effects in heterogeneous porous media. Computational Geosciences, 11(3):261-274, 2007.

[18] H. Holden, K. H. Karlsen, and K.-A. Lie. Operator splitting methods for degenerate convection-diffusion equations. I. Convergence and entropy estimates. In Stochastic processes, physics and geometry: new interplays, II (Leipzig, 1999), volume 29 of CMS Conf. Proc., pages 293-316. Amer. Math. Soc., Providence, RI, 2000.

[19] H. Holden, K. H. Karlsen, and N. H. Risebro. On uniqueness and existence of entropy solutions of weakly coupled systems of nonlinear degenerate parabolic equations. Electron. J. Differential Equations, pages No. 46, 31, 2003.

[20] K. H. Karlsen, N. H. Risebro, and E. B. Storrøsten. $L^{1}$ error estimates for difference approximations of degenerate convection-diffusion equations. Math. Comp., 83(290):2717-2762, 2014.

[21] K. H. Karlsen, N. H. Risebro, and E. B. Storrøsten. On the convergence rate of finite difference methods for degenerate convection-diffusion equations in several space dimensions. ESAIM: Mathematical Modelling and Numerical Analysis, pages 1-41, 2015 .

[22] K. H. Karlsen, N. H. Risebro, and J. D. Towers. On a nonlinear degenerate parabolic transport-diffusion equation with a discontinuous coefficient. Electron. J. Differential Equations, pages No. 93, 23, 2002.

[23] K. H. Karlsen, N. H. Risebro, and J. D. Towers. Upwind difference approximations for degenerate parabolic convection-diffusion equations with a discontinuous coefficient. IMA J. Numer. Anal., 22(4):623-664, 2002.

[24] S. N. Kružkov. First order quasilinear equations with several independent variables. Mat. Sb. (N.S.), 81 (123):228-255, 1970.

[25] A. Kurganov and E. Tadmor. New high-resolution central schemes for nonlinear conservation laws and convection-diffusion equations. J. Comput. Phys., 160(1):241-282, 2000.

[26] M. Ledoux and M. Talagrand. Probability in Banach spaces. Classics in Mathematics. Springer-Verlag, Berlin, 2011. Isoperimetry and processes, Reprint of the 1991 edition.

[27] L. Leibenzon. Complete Works, volume 2, chapter The Motion of a Gas in a Porous Medium. Acad. Sciences URSS, Moscow, 1930.

[28] S. Mishra, N. H. Risebro, C. Schwab, and S. Tokareva. Numerical solution of scalar conservation laws with random flux functions. SIAM/ASA Journal on Uncertainty Quantification, 4(1):552-591, 2016.

[29] S. Mishra and C. Schwab. Sparse tensor multi-level Monte Carlo finite volume methods for hyperbolic conservation laws with random initial data. Math. Comp., 81(280):1979-2018, 2012.

[30] S. Mishra, C. Schwab, and J. Šukys. Multi-level monte carlo finite volume methods for uncertainty quantification in nonlinear systems of balance laws. Journ. Comp. Phys., 2013. 
[31] M. Muskat and M. W. Meres. The flow of heterogeneous fluids through porous media. Physics, 7(9):346-363, 1936.

[32] M. Ohlberger. A posteriori error estimates for vertex centered finite volume approximations of convection-diffusion-reaction equations. M2AN Math. Model. Numer. Anal., 35(2):355-387, 2001.

[33] O. A. Oleŭnik and S. N. Kružkov. Quasi-linear parabolic second-order equations with several independent variables. Uspehi Mat. Nauk, 16(5 (101)):115-155, 1961.

[34] N. Risebro, C. Schwab, and F. Weber. Multilevel monte carlo front-tracking for random scalar conservation laws. BIT Numerical Mathematics, pages 1-30, 2015.

[35] J. Smoller. Shock waves and reaction-diffusion equations, volume 258 of Grundlehren der Mathematischen Wissenschaften [Fundamental Principles of Mathematical Sciences]. Springer-Verlag, New York, second edition, 1994.

[36] J. van Neerven. Stochastic evolution equations. Lecture Notes, ISEM, 2007/8.

[37] A. I. Volpert. Generalized solutions of degenerate second-order quasilinear parabolic and elliptic equations. Adv. Differential Equations, 5(10-12):1493-1518, 2000.

[38] A. I. Volpert and S. I. Hudjaev. The Cauchy problem for second order quasilinear degenerate parabolic equations. Mat. Sb. (N.S.), 78 (120):374-396, 1969.

[39] Y. B. Zel'dovich and Y. P. Raizer. Physics of Shock Waves and High-Temperature Hydrodynamic Phenomena, volume 2. Academic Press, New York, 1966. 